\title{
Two Parameter Families of Global Solutions for Some Classes of Nonlinear Second Order Ordinary Differential Equations
}

\author{
G. A. Grigorian \\ Institute of mathematics NAS of Armenia \\ Email: mathphys2@instmath.sci.am
}

\begin{abstract}
The Riccati equation method is used to establish some criteria for the existence of two-parameter families of global solutions for some classes of second order non-linear ordinary differential equations. Two oscillation theorems are proved. The results are applied to the Emden Fowler equation and to the Van der Pol type equation.
\end{abstract}

Keywords: Riccati equation, global solvability, oscillation, singular oscillation, Emden - Fowler equation, conditional stability, Van der Pol type equation.

\section{Introduction}

Due to numerous applications the nonlinear ordinary differential equations occupy an important place in the theory of differential equations and numerous works are devoted to them (see [1] and cited works in it, [2 - 8]). Except in rare cases these equations cannot be integrated explicitly. Therefore the study of such characteristics of their solutions as global existence, oscillation, asymptotic behavior, stability and plays an important role.

Let $p_{0}(t ; w), q_{0}(t ; w)$ and $r_{0}(t ; w)$ be continuous on $\left[t_{0} ;+\infty\right) \times(-\infty ;+\infty)$ real valued functions, and let $p_{0}(t ; w)>0, \quad t \geq t_{0}, w \in(-\infty ;+\infty)$. Consider the equation

$$
\left(p_{0}(t ; \phi(t)) \phi^{\prime}(t)\right)^{\prime}+q_{0}(t ; \phi(t)) \phi^{\prime}(t)+r_{0}(t ; \phi(t)) \phi(t)=0, \quad t \geq t_{0}
$$

Like the linear differential equations of the second order, this equation can be interpreted as a system of nonlinear differential equations of the first order (see [3], p. 381):

$$
\left\{\begin{array}{l}
\phi^{\prime}(t)=\frac{\psi(t)}{p_{0}(t ; \phi(t))} ; \\
\psi^{\prime}(t)=-r_{0}(t ; \phi(t)) \phi(t)-\frac{q_{0}(t ; \phi(t))}{p_{0}(t ; \phi(t))} \psi(t) .
\end{array}\right.
$$

By Peano's theorem (see [3], p. 21, 22) for every $\phi_{(0)}$ and $\phi_{(1)}$ (here and henceforth $\phi_{(0)}$ and $\phi_{(1)}$ are real numbers) and $t_{1} \geq t_{0}$ the system (1.2) has a solution $(\phi(t), \psi(t))$ in the neighborhood of the point $t_{1}$ (in the case $t_{1}=t_{0}$ in some right neighborhood of $\left.t_{0}\right)$, satisfying the initial conditions: $\phi\left(t_{1}\right)=\phi_{(0)}, \psi\left(t_{1}\right)=\phi_{(1)}$. Therefore, for any $\phi_{(0)}, \phi_{(1)}$ and $t_{1} \geq t_{0}$ eq. (1.1) has a solution in some neighborhood of the point $t_{1}$, satisfying the initial conditions: $\phi\left(t_{1}\right)=\phi_{(0)}, \phi^{\prime}\left(t_{1}\right)=\phi_{(1)}$.

Remark 1.1. The solution $\phi(t)$ of eq. (1.1), satisfying the initial conditions: $\phi\left(t_{1}\right)=\phi_{(0)}, \phi^{\prime}\left(t_{1}\right)=\phi_{(1)}$ in general, is not the unique. However, under additional restrictions on the functions $p_{0}(t ; w), q_{0}(t ; w)$ and $r_{0}(t ; w)$ it is the unique. For example, due to $(1.2)$, if the functions $f_{1}(t ; u ; v) \equiv \frac{v}{p_{0}(t ; u)}, f_{2}(t ; u ; v) \equiv$ $r_{0}(t ; u) u+\frac{q_{0}(t ; u)}{p_{0}(t ; u)} v$ satisfy the Lipschitz condition jointly $u, v$ in the region $O \equiv\left\{(t ; u ; v):\left|t-t_{1}\right| \leq\right.$ $\left.\delta,\left|u-\phi_{(0)}\right| \leq M,\left|v-\phi_{(1)}\right| \leq N\right\}, \quad \delta>0, \quad M>0, \quad N>0$ (see [3]. p. 13), then by virtue of the Picard Lindellef's theorem (see [3], p. 19) the solution $\phi(t)$ of eq. (1.1) with $\phi\left(t_{1}\right)=\phi_{(0)}, \phi^{\prime}\left(t_{1}\right)=\phi_{(1)}$ exists on the interval $\left[t_{1} ; t_{2}\right]$ and is the unique, where $t_{2} \equiv \min \left\{\delta, \frac{\sqrt{M^{2}+N^{2}}}{M_{0}}\right\}, M_{0} \equiv \max _{(t ; u ; v) \in O} \sqrt{f_{1}^{2}(t ; u ; v)+f_{2}^{2}(t ; u ; v)}$ (as far as the solution $(\phi(t), \psi(t))$ of the system $(1.2)$ with $\phi\left(t_{1}\right)=\phi_{(0)}, \quad \psi\left(t_{1}\right)=\phi_{(1)}$ exists on the interval $\left.\left[t_{1} ; t_{2}\right]\right)$. 
Example 1.1. For $p_{0}(t ; u) \equiv|u|^{\sigma}, \sigma>0, \quad q_{0}(t ; u) \equiv 0, \quad r_{0}(t ; u) \equiv|u|^{\nu}, \quad \nu<0$ the functions $f_{j}(t ; u ; v), \quad j=$ 1,2 , satisfy the Lipschtz condition in the region $\left|t-t_{1}\right| \leq \delta, \delta>0,|u-1| \leq \frac{1}{2},|v| \leq 1$, but not in the region $\left|t-t_{1}\right| \leq \delta, \quad \delta>0, \quad|u-1| \leq 2, \quad|v| \leq 1$

Example 1.2. For $p_{0}(t ; u) \equiv 1+t^{2}+u^{4}, q_{0}(t ; u) \equiv t+u^{3}, r_{0}(t ; u) \equiv t^{3}-u$ the functions $f_{j}(t ; u ; v), \quad j=$ 1,2 , satisfy the Lipschtz condition in the region $\left|t-t_{1}\right| \leq \delta,|u| \leq M,|v| \leq N, \delta>0, \quad M>0, \quad N>0$.

In this paper the Riccati equation method is applied to establish some criteria of existence of twoparameter family of global solutions of the equation (1.1). Two oscillatory theorems are proved. The obtained results are applied to Emden - Fowler's equation and to the Van der Pol type equation.

\section{Auxiliary Propositions}

Throughout this paragraph let $\phi_{0}(t)$ be a solution of eq.(1.1) on the interval $\left[t_{0} ; T\right)(T \leq+\infty) ; y_{0}(t) \equiv$ $p_{0}\left(t ; \phi_{0}(t)\right) \frac{\phi_{0}^{\prime}(t)}{\phi_{0}(t)}$. Consider the Riccati equation

$$
y^{\prime}(t)+\frac{y^{2}(t)}{p_{0}\left(t ; \phi_{0}(t)\right)}+\frac{q_{0}\left(t ; \phi_{0}(t)\right)}{p_{0}\left(t ; \phi_{0}(t)\right)} y(t)+r_{0}\left(t ; \phi_{0}(t)\right)=0, \quad t \in\left[t_{0} ; T\right) .
$$

Let $\phi_{0}(t) \neq 0, \quad t \in\left[t_{1} ; t_{2}\right)\left(\subset\left[t_{0} ; T\right)\right)$. It is not difficult to check, that the function $y_{0}(t)$ is a solution of eq. (2.1) on the interval $\left[t_{1} ; t_{2}\right)$. We have:

$$
\phi_{0}(t)=\phi_{0}\left(t_{1}\right) \exp \left\{\int_{t_{1}}^{t} \frac{\phi_{0}^{\prime}(\tau)}{\phi_{0}(\tau)} \mathrm{d} \tau\right\}=\phi_{0}\left(t_{1}\right) \exp \left\{\int_{t_{1}}^{t} \frac{y_{0}(\tau)}{p_{0}\left(\tau ; \phi_{0}(\tau)\right)} \mathrm{d} \tau\right\}, \quad t \in\left[t_{1} ; t_{2}\right) .
$$

Consider the linear equation

$$
z^{\prime}(t)+\frac{y_{0}(t)+q_{0}\left(t ; \phi_{0}(t)\right)}{p_{0}\left(t ; \phi_{0}(t)\right)} z(t)+r_{0}\left(t ; \phi_{0}(t)\right)=0, \quad t \in\left[t_{1} ; t_{2}\right)
$$

By virtue of the Cauchy's formula the general solution of this equation on the interval $\left[t_{1} ; t_{2}\right)$ is given by the formula

$$
\begin{aligned}
z(t)=c \exp \left\{-\int_{t_{1}}^{t} \frac{y_{0}(\tau)+q_{0}\left(\tau ; \phi_{0}(\tau)\right)}{p_{0}\left(\tau ; \phi_{0}(\tau)\right)} \mathrm{d} \tau\right\}- \\
\\
\quad-\int_{t_{1}}^{t} \exp \left\{-\int_{\tau}^{t} \frac{y_{0}(s)+q_{0}\left(s ; \phi_{0}(s)\right)}{p_{0}\left(s ; \phi_{0}(s)\right)} \mathrm{d} s\right\} r_{0}\left(\tau ; \phi_{0}(\tau)\right) \mathrm{d} \tau, \quad c=\text { const. }
\end{aligned}
$$

By $(2.1) y_{0}(t)$ is a solution of (2.3). Therefore from (2.4) it follows:

$$
\begin{aligned}
y_{0}(t)=y_{0}\left(t_{1}\right) \exp \{- & \left.\int_{t_{1}}^{t} \frac{y_{0}(\tau)+q_{0}\left(\tau ; \phi_{0}(\tau)\right)}{p_{0}\left(\tau ; \phi_{0}(\tau)\right)} \mathrm{d} \tau\right\}- \\
& -\int_{t_{1}}^{t} \exp \left\{-\int_{\tau}^{t} \frac{y_{0}(s)+q_{0}\left(s ; \phi_{0}(s)\right)}{p_{0}\left(s ; \phi_{0}(s)\right)} \mathrm{d} s\right\} r_{0}\left(\tau ; \phi_{0}(\tau)\right) \mathrm{d} \tau, \quad t \in\left[t_{1} ; t_{2}\right) .
\end{aligned}
$$

By (1.1) the following equality holds

$$
\left[p_{0}\left(t ; \phi_{0}(t)\right) \phi_{0}^{\prime}(t)\right]^{\prime}+\frac{q_{0}\left(t ; \phi_{0}(t)\right)}{p_{0}\left(t ; \phi_{0}(t)\right)}\left[p_{0}\left(t ; \phi_{0}(t)\right) \phi_{0}^{\prime}(t)\right]+r_{0}\left(t ; \phi_{0}(t)\right) \phi_{0}(t)=0, \quad t \geq t_{0}
$$


Therefore,

$$
\begin{aligned}
p_{0}\left(t ; \phi_{0}(t)\right) \phi_{0}^{\prime}(t)=p_{0}\left(t_{1} ; \phi_{0}\left(t_{1}\right)\right) \phi_{0}^{\prime}\left(t_{1}\right) \exp \left\{-\int_{t_{1}}^{t} \frac{q_{0}\left(\tau ; \phi_{0}(\tau)\right)}{p_{0}\left(\tau ; \phi_{0}(\tau)\right)} \mathrm{d} \tau\right\}- \\
\quad-\int_{t_{1}}^{t} \exp \left\{-\int_{\tau}^{t} \frac{q_{0}\left(s ; \phi_{0}(s)\right)}{p_{0}\left(s ; \phi_{0}(s)\right)} \mathrm{d} s\right\} r_{0}\left(\tau ; \phi_{0}(\tau)\right) \phi_{0}(\tau) \mathrm{d} \tau, \quad t \in\left[t_{0} ; T\right) .
\end{aligned}
$$

Dividing both sides of this equality on $p_{0}\left(t ; \phi_{0}(t)\right)$ and integrating from $t_{1}$ to $t$ we obtain:

$$
\begin{aligned}
\phi_{0}(t)= & \phi_{0}\left(t_{1}\right)+p_{0}\left(t_{1} ; \phi_{0}\left(t_{1}\right)\right) \phi_{0}^{\prime}\left(t_{1}\right) \int_{t_{1}}^{t} \exp \left\{-\int_{t_{1}}^{\tau} \frac{q_{0}\left(s ; \phi_{0}(s)\right)}{p_{0}\left(s ; \phi_{0}(s)\right)} \mathrm{d} s\right\} \frac{\mathrm{d} \tau}{p_{0}\left(\tau ; \phi_{0}(\tau)\right)}- \\
& -\int_{t_{1}}^{t} \frac{\mathrm{d} \tau}{p_{0}\left(\tau ; \phi_{0}(\tau)\right)} \int_{t_{1}}^{\tau} \exp \left\{-\int_{s}^{\tau} \frac{q_{0}\left(\xi ; \phi_{0}(\xi)\right)}{p_{0}\left(\xi ; \phi_{0}(\xi)\right)} \mathrm{d} \xi\right\} r_{0}\left(s ; \phi_{0}(s)\right) \phi_{0}(s) \mathrm{d} s .
\end{aligned}
$$

Let $p_{1}(t ; w), q_{1}(t ; w)$ and $r_{1}(t ; w)$ be real valued continuous functions on the $\left[t_{0} ;+\infty\right) \times(-\infty ;+\infty)$, and let $p_{1}(t ; w)>0, t \geq 0, w \in(-\infty ;+\infty)$. Along with the (1.1) consider the equation

$$
\left(p_{1}(t ; \phi(t)) \phi^{\prime}(t)\right)^{\prime}+q_{1}(t ; \phi(t)) \phi^{\prime}(t)+r_{1}(t ; \phi(t)) \phi(t)=0, \quad t \geq t_{0} .
$$

Throughout this paragraph let $\phi_{1}(t)$ be a solution of eq.(2.8) on the interval $\left[t_{0} ; T\right)(T \leq$ $\leq+\infty) ; y_{1}(t) \equiv p_{1}\left(t ; \phi_{1}(t)\right) \frac{\phi_{1}^{\prime}(t)}{\phi_{1}(t)}$. Consider the Riccati equation

$$
y^{\prime}(t)+\frac{y^{2}(t)}{p_{1}\left(t ; \phi_{1}(t)\right)}+\frac{q_{1}\left(t ; \phi_{1}(t)\right)}{p_{1}\left(t ; \phi_{1}(t)\right)} y(t)+r_{1}\left(t ; \phi_{1}(t)\right)=0, \quad t \in\left[t_{0} ; T\right) .
$$

Let $\phi_{1}(t) \neq 0, \quad t \in\left[t_{1} ; t_{2}\right)\left(\subset\left[t_{0} ; T\right)\right.$. Then as in the case of eq. (2.1) the function $y_{1}(t)$ is a solution of eq. (2.9) on the interval $\left[t_{1} ; t_{2}\right)$ and

$$
\phi_{1}(t)=\phi_{1}\left(t_{1}\right) \exp \left\{\int_{t_{1}}^{t} \frac{y_{1}(\tau)}{p_{1}\left(\tau ; \phi_{1}(\tau)\right)} \mathrm{d} \tau\right\}, \quad t \in\left[t_{1} ; t_{2}\right) .
$$

Since $y_{0}(t)$ and $y_{1}(t)$ are solutions of eq. (2.1) and eq. (2.9) respectively, we have:

$$
y_{j}^{\prime}(t)+\frac{y_{j}^{2}(t)}{p_{j}\left(t ; \phi_{j}(t)\right)}+\frac{q_{j}\left(t ; \phi_{j}(t)\right)}{p_{j}\left(t ; \phi_{1}(t)\right)} y_{j}(t)+r_{j}\left(t ; \phi_{j}(t)\right)=0, \quad j=0,1 .
$$

Therefore,

$$
\begin{aligned}
& {\left[y_{1}(t)-y_{0}(t)\right]^{\prime}+\frac{y_{0}(t)+y_{1}(t)+q_{1-j}\left(t ; \phi_{1-j}(t)\right)}{p_{1-j}\left(t ; \phi_{1-j}(t)\right)}\left[y_{1}(t)-y_{0}(t)\right]+} \\
& +\left[\frac{1}{p_{1}\left(t ; \phi_{1}(t)\right)}-\frac{1}{p_{0}\left(t ; \phi_{0}(t)\right)}\right] y_{j}^{2}(t)+\left[\frac{q_{1}\left(t ; \phi_{1}(t)\right)}{p_{1}\left(t ; \phi_{1}(t)\right)}-\frac{q_{0}\left(t ; \phi_{0}(t)\right)}{p_{0}\left(t ; \phi_{0}(t)\right)}\right] y_{j}(t)+ \\
& \quad+r_{1}\left(t ; \phi_{1}(t)\right)-r_{0}\left(t ; \phi_{0}(t)\right)=0, \quad t \in\left[t_{1} ; t_{2}\right), \quad j=0,1 .
\end{aligned}
$$

By (2.5) from (2.11) we have:

$$
y_{1}(t)-y_{0}(t)=\left[y_{1}\left(t_{1}\right)-y_{0}\left(t_{1}\right)\right] \exp \left\{-\int_{t_{1}}^{t} \frac{y_{0}(\tau)+y_{1}(\tau)+q_{1-j}\left(\tau ; \phi_{1-j}(\tau)\right)}{p_{1-j}\left(\tau ; \phi_{1-j}(\tau)\right)} \mathrm{d} \tau\right\}-
$$




$$
\begin{aligned}
& -\int_{t_{1}}^{t} \exp \left\{-\int_{\tau}^{t} \frac{y_{0}(s)+y_{1}(s)+q_{1-j}\left(s ; \phi_{1-j}(s)\right)}{p_{1-j}\left(s ; \phi_{1}(s)\right)} \mathrm{d} s\right\}\left[\left(\frac{1}{p_{1}\left(\tau ; \phi_{1}(\tau)\right)}-\frac{1}{p_{0}\left(\tau ; \phi_{0}(\tau)\right)}\right) y_{j}^{2}(\tau)+\right. \\
& \left.+\left(\frac{q_{1}\left(\tau ; \phi_{1}(\tau)\right)}{p_{1}\left(\tau ; \phi_{1}(\tau)\right)}-\frac{q_{0}\left(\tau ; \phi_{0}(\tau)\right)}{p_{0}\left(\tau ; \phi_{0}(\tau)\right)}\right) y_{j}(\tau)+r_{1}\left(\tau ; \phi_{1}(\tau)\right)-r_{0}\left(\tau ; \phi_{0}(\tau)\right)\right] \mathrm{d} \tau, \quad j=0,1 .
\end{aligned}
$$

Lemma 2.1. Let $y_{0}\left(t_{1}\right) \geq 0$, and let the inequality

$$
r_{0}(t ; w) \leq 0 \quad \text { for } \quad 0<w \leq Y_{0}(t), \quad t \in\left[t_{1} ; t_{2}\right),
$$

holds, where $Y_{0}(t) \equiv \max _{\xi \in\left[t_{1} ; t\right]}\left|\phi_{0}(\xi)\right|$. Then

$$
y_{0}(t) \geq 0, \quad t \in\left[t_{1} ; t_{2}\right),
$$

and if $y_{0}\left(t_{1}\right)>0$, then

$$
y_{0}(t)>0, \quad t \in\left[t_{1} ; t_{2}\right),
$$

Proof. It follows from (2.13), that $r_{0}(t, \phi(t)) \leq 0, t \in\left[t_{1} ; t_{2}\right)$. Then

$$
\int_{t_{1}}^{t} \exp \left\{-\int_{\tau}^{t} \frac{y_{0}(s)+q_{0}\left(s, \phi_{0}(s)\right)}{p_{0}\left(s, \phi_{0}(s)\right)} \mathrm{d} s\right\} r_{0}\left(\tau, \phi_{0}(\tau)\right) \mathrm{d} \tau \leq 0, \quad t \in\left[t_{1} ; t_{2}\right) .
$$

From here, from (2.5) and from the inequality $y_{0}\left(t_{1}\right) \geq 0\left(y_{0}\left(t_{1}\right)>0\right)$ it follows $(2.14)((2.15))$. The proof of the lemma is complete.

Definition 2.1. We say, that $\left[t_{1} ; t_{2}\right)$ is maximum interval of existence for the solution $\phi_{0}(t)\left(y_{0}(t), \phi_{1}(t), y_{1}(t)\right)$, if $\phi_{0}(t)\left(y_{0}(t), \phi_{1}(t), y_{1}(t)\right)$ exists on the interval $\left[t_{1} ; t_{2}\right)$ and cannot be continued to right of $t_{2}$ as a solution of eq. (1.1) $((2.1),(2.8),(2.9))$.

Lemma 2.2. Let $y_{0}(t) \geq 0, t \in\left[t_{1} ; t_{2}\right)$, and let

$$
\int_{t_{1}}^{t_{2}} \frac{y_{0}(\tau)}{p_{0}\left(\tau ; \phi_{0}(\tau)\right)} \mathrm{d} \tau<+\infty
$$

Then $\left[t_{1} ; t_{2}\right)$ is not maximum interval of existence for $y_{0}(t)$.

Proof. Since $y_{0}(t)$ is nonnegative, it follows from (2.2) and (2.16), that there exists finite limit

$$
\lim _{t \rightarrow t_{2}-0} \phi_{0}(t)=\phi_{0}\left(t_{1}\right) \exp \left\{\lim _{t \rightarrow t_{2}-0} \int_{t_{1}}^{t} \frac{y_{0}(\tau)}{p_{0}\left(\tau ; \phi_{0}(\tau)\right)} \mathrm{d} \tau\right\} \neq 0 .
$$

By (2.6) from here and from continuity of $p_{0}(t ; w), q_{0}(t ; w), r_{0}(t ; w)$ it follows existence of finite limit $\lim _{t \rightarrow t_{2}-0} \phi_{0}^{\prime}(t)$. Therefore, $\left[t_{1} ; t_{2}\right)$ is not maximum interval of existence for $\phi_{0}(t)$, and, so, by virtue of $(2.17)$ the function $p_{0}\left(t ; \phi_{0}(t)\right) \frac{\phi_{0}^{\prime}(t)}{\phi_{0}(t)}$ is defined on the interval $\left[t_{1} ; t_{2}+\varepsilon\right)$ for some $\varepsilon>0$. It follows from here, that $\left[t_{1} ; t_{2}\right)$ is not maximum interval of existence for $y_{0}(t)$. The proof of the lemma is completed.

Let $u=u(t) \neq 0, v=v(t), \quad x=x(t)$ be continuous functions on the interval $\left[t_{0} ;+\infty\right)$,

$$
I_{u, v}^{+}\left(t_{1} ; t\right) \equiv \int_{t_{1}}^{t} \exp \left\{-\int_{t_{1}}^{\tau} v(s) \mathrm{d} s\right\} \frac{\mathrm{d} \tau}{u(\tau)}, \quad I_{v, x}^{-}\left(t_{1} ; t\right) \equiv \int_{t_{1}}^{t} \exp \left\{-\int_{\tau}^{t} v(s) \mathrm{d} s\right\} x(\tau) \mathrm{d} \tau,
$$

$P(t), Q(t)$ and $R(t)$ be real valued continuous functions on the interval $\left[t_{0} ;+\infty\right)$, and let $P(t)>0, t \geq t_{0}$. Denote:

$$
F\left(t_{1} ; t ; c_{1} ; c_{2}\right) \equiv\left|c_{1}\right| \exp \left\{c_{2} I_{P, Q}^{+}\left(t_{1} ; t\right)-\int_{t_{1}}^{t} I_{Q, R}^{-}\left(t_{1} ; \tau\right) \frac{\mathrm{d} \tau}{P(\tau)}\right\}, t_{1}, \quad t \geq t_{0} .
$$


Lemma 2.3. Let $y_{0}(t) \geq 0, t \in\left[t_{1} ; t_{2}\right)$, and let for some $\varepsilon>0$ the following inequalities hold:

$$
p_{0}(t ; w) \geq P(t), \frac{q_{0}(t ; w)}{p_{0}(t ; w)} \geq Q(t), \quad R(t) \leq r_{0}(t ; w) \leq 0
$$

for $|w| \leq F\left(t_{1} ; t ; c_{1} ; c_{2}\right)+\varepsilon, \quad t \in\left[t_{1} ; t_{2}\right)$, where $c_{1} \equiv \phi_{0}\left(t_{1}\right) \neq 0, c_{2} \equiv y_{0}\left(t_{1}\right)$. Then

$$
\int_{t_{1}}^{t} \frac{y_{0}(\tau)}{p_{0}\left(\tau ; \phi_{0}(\tau)\right)} \mathrm{d} \tau \leq y_{0}\left(t_{1}\right) I_{P, Q}^{+}\left(t_{1} ; t\right)-\int_{t_{1}}^{t} I_{Q, R}^{-}\left(t_{1} ; \tau\right) \frac{\mathrm{d} \tau}{P(\tau)}, \quad t \in\left[t_{1} ; t_{2}\right) .
$$

Proof. Suppose, that for some $t_{3} \in\left(t_{1} ; t_{2}\right)$ the inequality $(2.19)$ is not true. Then because this inequality holds for $t=t_{1}$, then taking into account $(2.2)$ we get, that there exists $t_{4} \in\left(t_{1} ; t_{3}\right)$ such, that

$$
\begin{gathered}
\left|\phi_{0}\left(t_{4}\right)\right|>F\left(t_{1} ; t_{4} ; c_{1} ; c_{2}\right) ; \\
\left|\phi_{0}(t)\right| \leq F\left(t_{1} ; t ; c_{1} ; c_{2}\right)+\varepsilon, \quad t \in\left[t_{1} ; t_{4}\right) .
\end{gathered}
$$

From the last inequality and from(2.20) it follows, that

$$
p_{0}\left(t ; \phi_{0}(t)\right) \geq P(t), \frac{q_{0}\left(t ; \phi_{0}(t)\right)}{p_{0}\left(t ; \phi_{0}(t)\right)} \geq Q(t) ; \quad R(t) \leq r_{0}\left(t ; \phi_{0}(t)\right) \leq 0, \quad t \in\left[t_{1} ; t_{4}\right] .
$$

By virtue of nonnegativity of $y_{0}(t)$ from here and (2.5) it follows:

$$
\frac{y_{0}(t)}{p_{0}\left(t ; \phi_{0}(t)\right)} \leq \frac{c_{2}}{P(t)} \exp \left\{-\int_{t_{1}}^{t} Q(\tau) \mathrm{d} \tau\right\}-\frac{1}{P(t)} I_{Q, R}^{-}\left(t_{1} ; t\right) ; \quad t \in\left[t_{1} ; t_{4}\right] .
$$

Integrate this inequality from $t_{1}$ to $t_{4}$. We obtain:

$$
\int_{t_{1}}^{t_{4}} \frac{y_{0}(\tau)}{p_{0}\left(\tau ; \phi_{0}(\tau)\right)} \mathrm{d} \tau \leq c_{2} I_{P, Q}^{+}\left(t_{1} ; t_{4}\right)-\int_{t_{1}}^{t_{4}} I_{Q, R}^{-}\left(t_{1} ; \tau\right) \frac{\mathrm{d} \tau}{P(\tau)} .
$$

Consequently, $\left|\phi_{0}\left(t_{4}\right)\right| \leq F\left(t_{1} ; t_{4} ; c_{1} ; c_{2}\right)$, which contradicts $(2.20)$. The obtained contradiction proves (2.19). The proof of the lemma is complete.

Denote: $G_{x}\left(t_{1} ; t ; c_{1} ; c_{2}\right) \equiv\left|c_{1}\right| \exp \left\{c_{2} I_{P, Q}^{+}\left(t_{1} ; t\right)+\int_{t_{1}}^{t} \frac{x(\tau)}{P(\tau)} \mathrm{d} \tau\right\}$.

Lemma 2.4. Let $y_{0}(t) \geq 0, t \in\left[t_{1} ; t_{2}\right)$, and let for some $\varepsilon>0$ the following inequalities hold:

$$
p_{0}(t ; w) \geq P(t), \quad q_{0}(t ; w) \geq Q(t) \geq 0, \quad r_{0}(t ; w) \leq 0, \quad\left|p_{0}(t ; w) \frac{r_{0}(t ; w)}{q_{0}(t ; w)}\right| \leq \widetilde{Q}(t) \quad \text { for } \quad|w| \leq
$$

$\leq G_{M}\left(t_{1} ; t ; c_{1} ; c_{2}\right)+\varepsilon, t \in\left[t_{1} ; t_{2}\right)$, where $\widetilde{Q}(t)$ is a continuous function on the interval $\left[t_{1} ; t_{2}\right), M(t) \equiv$ $\max _{\xi \in\left[t_{1} ; t\right]}\{\widetilde{Q}(\xi)\}, \quad c_{1}=\phi_{0}\left(t_{1}\right), \quad c_{2}=y_{0}\left(t_{1}\right)$. Then

$$
\int_{t_{1}}^{t} \frac{y_{0}(\tau)}{p_{0}\left(\tau ; \phi_{0}(\tau)\right)} \mathrm{d} \tau \leq y_{0}\left(t_{1}\right) I_{P, Q}^{+}\left(t_{1} ; t\right)-\int_{t_{1}}^{t} \frac{M(\tau)}{P(\tau)} \mathrm{d} \tau, \quad t \in\left[t_{1} ; t_{2}\right) .
$$

This lemma can be proved by analogy of the lemma 2.3. In its proof can be used the following easily verifiable inequality

$$
\int_{t_{1}}^{t} \exp \left\{-\int_{\tau}^{t} \frac{y_{0}(s)+q_{0}\left(s ; \phi_{0}(s)\right)}{p_{0}\left(s ; \phi_{0}(s)\right)}\right\}\left|r_{0}\left(\tau ; \phi_{0}(\tau)\right)\right| \mathrm{d} \tau \leq M(t), \quad t \in\left[t_{1} ; t_{2}\right) .
$$


Lemma 2.5. Let the following conditions hold:

a) $y_{1}\left(t_{1}\right)>y_{0}\left(t_{1}\right)$;

$\left.\mathrm{b}_{1}\right) \phi_{1}\left(t_{1}\right) \geq \phi_{0}\left(t_{1}\right)>0, \quad \phi_{1}^{\prime}\left(t_{1}\right)>\phi_{0}^{\prime}\left(t_{1}\right) \geq 0$ or $\phi_{1}\left(t_{1}\right) \leq \phi_{0}\left(t_{1}\right)<0, \quad \phi_{1}^{\prime}\left(t_{1}\right)<\phi_{0}^{\prime}\left(t_{1}\right) \leq 0$

$\left.c_{1}\right) \quad p_{0}(t ; w) \leq p_{1}\left(t ; w_{1}\right), \quad r_{0}(t ; w) \geq r_{1}\left(t ; w_{1}\right)$, for $t \in\left[t_{1} ; t_{2}\right), \quad|w| \leq\left|w_{1}\right|, \quad w$, $w_{1} \in(-\infty ;+\infty)$

$\left.\mathrm{d}_{1}\right) p_{0}(t ; w) \geq p_{1}\left(t ; w_{1}\right)$ for $t \in\left[t_{1} ; t_{2}\right),|w| \geq\left|w_{1}\right|, w, w_{1} \in(-\infty ;+\infty)$;

e 1$) \frac{q_{0}(t ; w)}{p_{0}(t ; w)} \leq \frac{q_{1}\left(t ; w_{1}\right)}{p_{1}\left(t ; w_{1}\right)} ; y_{1}(t) \geq 0$ or $y_{0}(t) \geq 0$ for $t \in\left[t_{1} ; t_{2}\right),|w| \leq\left|w_{1}\right|$, $w, w_{1} \in(-\infty ;+\infty)$

Then

$$
y_{1}(t)>y_{0}(t), \quad t \in\left[t_{1} ; t_{2}\right) .
$$

Proof. Suppose (2.23) is false. Then from $\left.\mathrm{a}_{1}\right)$ it follows:

$$
\begin{gathered}
y_{1}(t)>y_{0}(t), \quad t \in\left[t_{1} ; t_{3}\right) ; \\
y_{1}\left(t_{3}\right)=y_{0}\left(t_{3}\right)
\end{gathered}
$$

for some $t_{3} \in\left(t_{1} ; t_{2}\right)$. From $\left.b_{1}\right)$ it follows:

$$
\left|\phi_{1}(t)\right| \geq\left|\phi_{0}(t)\right|, \quad t \in\left[t_{1} ; t_{4}\right),
$$

for some $t_{4} \in\left[t_{1} ; t_{3}\right]$. Let us show, that

$$
\left|\phi_{0}(t)\right| \leq\left|\phi_{1}(t)\right|, \quad t \in\left[t_{1} ; t_{3}\right) .
$$

Suppose, that it is not so. Then it follows from (2.24), that

$$
\begin{aligned}
& \left|\phi_{0}(t)\right| \leq\left|\phi_{1}(t)\right|, \quad t \in\left[t_{1} ; t_{5}\right], \\
& \left|\phi_{0}(t)\right|>\left|\phi_{1}(t)\right|, \quad t \in\left(t_{5} ; t_{3}\right),
\end{aligned}
$$

where $t_{5}=\sup \left\{t \in\left[t_{1} ; t_{3}\right):\left|\phi_{0}(t)\right| \leq\left|\phi_{1}(t)\right|\right\} \in\left(t_{1} ; t_{3}\right)$. On the strength of $(2.2)$ and $(2.10)$ from $\left.\mathrm{d}_{1}\right)$, (2.23), (2.26) and (2.27) it follows:

$$
\left|\phi_{0}(t)\right|=\left|\phi_{0}\left(t_{5}\right)\right| \exp \left\{\int_{t_{1}}^{t} \frac{y_{0}(\tau)}{p_{0}\left(\tau ; \phi_{0}(\tau)\right)} \mathrm{d} \tau\right\} \leq\left|\phi_{1}\left(t_{5}\right)\right| \exp \left\{\int_{t_{1}}^{t} \frac{y_{1}(\tau)}{p_{1}\left(\tau ; \phi_{1}(\tau)\right)} \mathrm{d} \tau\right\}=\left|\phi_{1}(t)\right|,
$$

$t \in\left[t_{5} ; t_{3}\right)$, which contradicts (2.27). The obtained contradiction proves (2.25). By (2.12) from $\left.\left.\mathrm{c}_{1}\right), \mathrm{e}_{1}\right)$ and (2.25) it follows, that $y_{1}\left(t_{3}\right)>y_{0}\left(t_{3}\right)$, which contradicts $(2.23)$. The obtained contradiction proves (2.21). The proof of the lemma is complete.

Remark 2.1. It follows from the conditions $\left.c_{1}\right)$ and $\left.d_{1}\right)$, that $p_{0}(t ; w)=p_{1}(t ; w), t \in \in\left[t_{1} ; t_{2}\right), w \in$ $(-\infty ;+\infty)$ and $p_{0}(t ; w)$ increases (in the wide sense) by $w$ on the interval $[0 ;+\infty)$ and decreases (in the wide sense) by $w$ on the interval $(-\infty ; 0]$ for every $t \in\left[t_{1} ; t_{2}\right)$, in particular when $p_{0}(t ; w)=p_{1}(t ; w)=$ $p(t), \quad t \in\left[t_{1} ; t_{2}\right)$, then the conditions $\left.B_{1}\right)$ and $\left.r_{1}\right)$ hold.

Let us consider the Riccati equation

$$
y^{\prime}(t)+\frac{y^{2}(t)}{P(t)}+\frac{Q(t)}{P(t)} y(t)+R(t)=0, \quad t \geq t_{0} .
$$

Lemma 2.6. Let the following conditions hold:

a $) \quad y_{0}(t) \geq 0, \quad t \in\left[t_{1} ; t_{2}\right)\left(\subset\left[t_{0} ; T\right)\right)$;

$\left.\mathrm{b}_{2}\right) \quad P(t) \geq p_{0}\left(t ; \phi_{0}(t)\right), \quad \frac{Q(t)}{P(t)} \leq \frac{q_{0}\left(t ; \phi_{0}(t)\right)}{p_{0}\left(t ; \phi_{0}(t)\right)}, \quad R(t) \leq r_{0}\left(t ; \phi_{0}(t)\right), \quad t \in\left[t_{1} ; t_{2}\right)$.

Then the solution $y_{2}(t)$ of eq. (2.28), satisfying the condition $y_{2}\left(t_{1}\right) \geq y_{0}\left(t_{1}\right)$, exists on the interval $\left[t_{1} ; t_{2}\right)$.

Proof. Let $\left[t_{1} ; t_{3}\right)$ be the maximum interval of existence for $y_{2}(t)$. It is to show, that $t_{3} \geq t_{2}$. Suppose $t_{3}<t_{2}$. Then taking into account the inequality $y_{2}\left(t_{1}\right) \geq y_{0}\left(t_{1}\right)$, and $\left(2.12_{0}\right)$ we conclude: it follows from $\left.\mathrm{a}_{2}\right)$ and $\left.\mathrm{b}_{2}\right)$, that for $p_{1}(t ; w) \equiv P(t), q_{1}(t ; w) \equiv Q(t), \quad r_{1}(t ; w) \equiv R(t)$ the inequality $y_{2}(t) \geq y_{0}(t), \quad t \in\left[t_{1} ; t_{3}\right)$, 
holds. Since $y_{0}(t)$ is continuous on the interval $\left[t_{1} ; t_{3}\right]$, it follows from the last inequality, that the function $f(t) \equiv \int_{t_{1}}^{t} \frac{y_{2}(\tau)}{P(\tau)} \mathrm{d} \tau$ is bounded from below on the interval $\left[t_{1} ; t_{3}\right)$. It follows from here (see $[9$, p. 3 , the lemma 2.2]), that $\left[t_{1} ; t_{3}\right)$ is not maximum interval of existence for $y_{2}(t)$. The obtained contradiction shows, that $t_{3} \geq t_{2}$. The proof of the lemma is complete.

Lemma 2.7. Let $r_{0}(t ; w) \geq 0, t \in\left[T_{0} ; T\right), w \in(-\infty ;+\infty)\left(t_{0} \leq T_{0}<T \leq+\infty\right)$,

$\phi_{0}\left(T_{1}\right)=0$ for some $T_{1} \in\left[T_{0} ; T\right)$, and let $\sup _{t \in\left[T_{0} ; T\right)}\left|\phi_{0}(t)\right|>0$. Then $\phi_{0}(t)$ changes its sign on the interval $\left[T_{0} ; T\right)$.

Proof. Since $\sup _{t \in\left[T_{0} ; T\right)}\left|\phi_{0}(t)\right|>0$, there exists $T_{2} \in\left[T_{0} ; T\right)$ such, that $\phi_{0}\left(T_{2}\right) \neq 0$. Let $T_{2}<T_{1}$ (the proof in the case $T_{2}>T_{1}$ by analogy). Then there exists $T_{3} \in\left(T_{2} ; T_{1}\right]$ such, that

$$
\begin{gathered}
\phi_{0}\left(T_{3}\right)=0, \\
\phi_{0}(t) \neq 0, \quad t \in\left[T_{2} ; T_{3}\right) .
\end{gathered}
$$

By Lagrange's mean value theorem it follows from $(2.29)$ the existence of a $\xi \in\left(T_{2} ; T_{3}\right)$ such, that $\phi_{0}^{\prime}(\xi)=-\frac{\phi_{0}\left(T_{2}\right)}{T_{3}-T_{2}}$. So,

$$
\operatorname{sign} \phi_{0}^{\prime}(\xi)=-\operatorname{sign} \phi_{0}\left(T_{2}\right) \text {. }
$$

To complete the proof of the lemma it is enough to show, that

$$
\operatorname{sign} \phi_{0}^{\prime}\left(T_{3}\right)=-\operatorname{sign} \phi_{0}\left(T_{2}\right) \text {. }
$$

It follows from (2.30), that $y_{0}(t)$ exists on the interval $\left[T_{2} ; T_{3}\right)$. Therefore, by virtue of $(2.5)$ it follows from the nonnegativity of $r_{0}(t ; w)$, that sign $y_{0}\left(T_{3}\right)=$ sign $y_{0}(\xi)$. From here and $(2.31)$ it follows $(2.32)$. The proof of the lemma is complete.

\section{Some Global Solvability and Oscillatory Criteria}

Let $P(t), Q(t)$ and $R(t)$ be the same functions as in the previous section.

Theorem 3.1. Let $\phi_{(0)} \neq 0, \frac{\phi_{(1)}}{\phi_{(0)}} \geq 0$, and let for some $\varepsilon>0$ the following inequalities hold

$$
p_{0}(t ; w) \geq P(t), \frac{q_{0}(t ; w)}{p_{0}(t ; w)} \geq Q(t), \quad R(t) \leq r_{0}(t ; w) \leq 0
$$

for $|w| \leq F\left(t_{0} ; t ; \phi_{(0)} ; p\left(t_{0} ; \phi_{(0)}\right) \frac{\phi_{(1)}}{\phi_{(0)}}\right)+\varepsilon, t \geq t_{0}$. Then the solution $\phi_{0}(t)$ of eq. (1.1), satisfying the initial value conditions: $\phi_{0}\left(t_{0}\right)=\phi_{(0)}, \phi_{0}\left(t_{0}\right)=\phi_{(1)}$, exists on the interval $\left[t_{0} ;+\infty\right)$. The function $\left|\phi_{0}(t)\right|$ is positive, nondecreasing and satisfies the estimate

$$
\left|\phi_{0}(t)\right| \leq F\left(t_{0} ; t ; \phi_{(0)} ; p_{0}\left(t_{0} ; \phi_{(0)}\right) \frac{\phi_{(1)}}{\phi_{(0)}}\right), \quad t \geq t_{0} .
$$

In this case if $\phi_{(1)} \neq 0$, then

$$
\phi^{\prime}(t) \neq 0, \quad t \geq t_{0}
$$

Proof. Let $\phi_{(0)}>0$ (the proof in the case $\phi_{(0)}<0$ by analogy), $\phi_{0}(t)$ be the solution of eq. (1.1), satisfying the initial value conditions: $\phi_{0}\left(t_{0}\right)=\phi_{(0)}, \phi_{0}^{\prime}\left(t_{0}\right)=\phi_{(1)}$ (existence of $\phi_{0}(t)$ follows from the connection between (1.1) and (1.2) and from the Peano's theorem; see [3, p. 21, the theorem 2.1]), and let $\left[t_{0} ; T\right.$ ) be the maximum interval of existence for $y_{0}(t) \equiv p_{0}\left(t ; \phi_{0}(t)\right) \frac{\phi_{0}^{\prime}(t)}{\phi_{0}(t)}$ (it assumes, that $\phi_{0}(t)$ exists on the interval $\left[t_{0} ; T\right)$ and does not vanish on it). Let us show, that

$$
y_{0}(t) \geq 0, \quad t \in\left[t_{0} ; T\right) .
$$


Suppose, that it is not so. Then since $y_{0}\left(t_{0}\right)=p_{0}\left(t_{0} ; \phi_{(0)}\right) \frac{\phi_{(1)}}{\phi_{(0)}} \geq 0$, there exists $\widetilde{t}_{0}, \widetilde{t}_{1}$ such, that

$$
\begin{aligned}
& y_{0}(t) \geq 0, \quad t \in\left[t_{0} ; \widetilde{t}_{0}\right], \\
& y_{0}(t)<0, \quad t \in\left(\widetilde{t}_{0} ; \widetilde{t}_{1}\right) .
\end{aligned}
$$

By (3.1) for $c_{1}=\phi_{(0)}, \quad c_{2}=p_{0}\left(t_{0} ; \phi_{(0)}\right) \frac{\phi_{(1)}}{\phi_{(0)}}, t_{1}=t_{0}$ the inequalities $(2.20)$ of the lemma 2.3 hold. Therefore taking into account $(2.2)$ we will have:

$$
\phi_{0}(t) \leq F\left(t_{0} ; t ; \phi_{(0)} ; p_{0}\left(t_{0} ; \phi_{(0)}\right) \frac{\phi_{(1)}}{\phi_{(0)}}\right), \quad t \in\left[t_{0} ; \widetilde{t}_{0}\right]
$$

By (2.2) from here and from (3.6) it follows:

$$
\widetilde{M}(t) \equiv \max _{\xi \in\left[t_{0} ; t\right]} \phi_{0}(\xi) \leq F\left(t_{0} ; t ; \phi_{(0)} ; p_{0}\left(t_{0} ; \phi_{(0)}\right) \frac{\phi_{(1)}}{\phi_{(0)}}\right), \quad t \in\left[t_{0} ; \widetilde{t}_{1}\right) .
$$

Then taking into account third of the inequalities (3.1) we will have: $r_{0}(t ; w) \leq 0$ for $0<w \leq \widetilde{M}(t), t \in$ $\left[t_{0} ; \widetilde{t}_{1}\right)$. On the strength of the lemma 2.1 we conclude from here, that $y_{0}(t) \geq 0$ for $t \in\left[t_{0} ; \widetilde{t}_{1}\right)$, which contradicts (3.6). The obtained contradiction proves (3.4). Let us show, that $T=+\infty$. Suppose $T<+\infty$. By virtue of the lemma 2.3 from (3.1) and (3.3) it follows:

$$
\int_{t_{0}}^{t} \frac{y_{0}(\tau)}{p_{0}\left(\tau ; \phi_{0}(\tau)\right)} \mathrm{d} \tau \leq y_{0}\left(t_{0}\right) I_{P, Q}^{+}\left(t_{0} ; t\right)-\int_{t_{0}}^{t} I_{Q, R}^{-}\left(t_{0} ; \tau\right) \frac{\mathrm{d} \tau}{P(\tau)}, \quad t \in\left[t_{0} ; T\right) .
$$

Therefore, $\int_{t_{0}}^{T} \frac{y_{0}(\tau)}{p_{0}\left(\tau ; \phi_{0}(\tau)\right)} \mathrm{d} \tau<+\infty$. On the strength of the lemma 2.2 it follows from here, that $\left[t_{0} ; T\right)$ is not maximal interval of existence for $y_{0}(t)$. The obtained contradiction shows, that $T=+\infty$. So $(3.7)$ is valid for all $t \geq t_{0}$. Therefore, by (2.2) the inequality $(3.2)$ holds. Since $\phi_{0}\left(t_{0}\right)=\phi_{(0)}>0$, and $y_{0}(t)$ is nonnegative, by $(2.2)$ the function $\phi_{0}(t)$ is positive and nondecreasing on the interval $\left[t_{0} ;+\infty\right)$. And if $\phi_{(1)}>0$, then by (2.2) and the lemma 2.1 the inequality (3.3) holds. The proof of the theorem is complete. Remark 3.1. A solution $\phi_{*}(t)$ of the equation

$$
\left(P(t) \phi^{\prime}(t)\right)^{\prime}+Q(t) \phi^{\prime}(t)+R(t) \phi(t)=0, \quad, t \geq t_{o},
$$

such, that $\phi_{*}(t) \neq 0, t \in\left[t_{1} ; t_{2}\right)$, is connected with the function $F$ by the following relation

$$
\begin{aligned}
&\left|\phi_{*}\left(t_{1}\right)\right| \exp \left\{\frac{\phi_{*}(t)}{\phi_{*}\left(t_{1}\right)}\right\}=F\left(t_{1} ; t ; \phi_{*}\left(t_{1}\right) ; P\left(t_{1}\right) \frac{\phi_{*}^{\prime}\left(t_{1}\right)}{\phi_{*}\left(t_{1}\right)}\right) \exp \left\{1-P\left(t_{1}\right) \frac{\phi_{*}^{\prime}\left(t_{1}\right)}{\phi_{*}\left(t_{1}\right)} \times\right. \\
&\left.\times \int_{t_{1}}^{t} \frac{\mathrm{d} \tau}{P(\tau)} \int_{t_{1}}^{\tau} \exp \left\{-\int_{s}^{\tau} \frac{Q(\xi)}{P(\xi)} d \xi\right\} R(s) I_{P, Q}^{+}\left(t_{1} ; s\right) \mathrm{d} s+\frac{1}{\phi_{*}\left(t_{1}\right)}\left(K^{2} \phi_{*}\right)(t)\right\},
\end{aligned}
$$

where $K$ - is the integral operator

$$
\left(K \phi_{*}\right)(t) \equiv \int_{t_{1}}^{t} \frac{\mathrm{d} \tau}{P(\tau)} \int_{t_{1}}^{\tau} \exp \left\{-\int_{s}^{\tau} \frac{Q(\xi)}{P(\xi)} d \xi\right\} R(s) \phi_{*}(s) \mathrm{d} s, \quad, t \in\left[t_{1} ; t_{2}\right) .
$$

Indeed, since $y_{*}(t) \equiv P(t) \frac{\phi_{*}^{\prime}(t)}{\phi_{*}(t)}$ is a solution of eq. (2.8) on the interval $\left[t_{1} ; t_{2}\right)$, by the Cauchy's formula

$$
y_{*}(t)=y_{*}\left(t_{1}\right) \exp \left\{-\int_{t_{1}}^{t} \frac{Q(\tau)}{P(\tau)} \mathrm{d} \tau-\int_{t_{1}}^{t} \frac{y_{*}(\tau)}{P(\tau)} \mathrm{d} \tau\right\}-
$$




$$
-\int_{t_{1}}^{t} \exp \left\{-\int_{\tau}^{t} \frac{Q(s)}{P(s)} \mathrm{d} s-\int_{\tau}^{t} \frac{y_{*}(s)}{P(s)} \mathrm{d} s\right\} R(\tau) \mathrm{d} \tau, \quad t \in\left[t_{1} ; t_{2}\right)
$$

Multiplying both sides of this equality on $\frac{1}{P(t)} \exp \left\{\int_{t_{1}}^{t} \frac{y_{*}(\tau)}{P(\tau)} \mathrm{d} \tau\right\}$ and integrating from $t_{1}$ to $t$ taking into account the equality $\phi_{*}(t)=\phi_{*}\left(t_{1}\right) \exp \left\{\int_{t_{1}}^{t} \frac{y_{*}(\tau)}{P(\tau)} \mathrm{d} \tau\right\}, t \in\left[t_{1} ; t_{2}\right)$, we obtain:

$$
\frac{\phi_{*}(t)}{\phi_{*}\left(t_{1}\right)}=1+P\left(t_{1}\right) \frac{\phi_{*}^{\prime}\left(t_{1}\right)}{\phi_{*}\left(t_{1}\right)} I_{P, Q}^{+}\left(t_{1} ; t\right)-\frac{1}{\phi_{*}\left(t_{1}\right)}\left(K \phi_{*}\right)(t), \quad t \in\left[t_{1} ; t_{2}\right) .
$$

After making the first iteration in this equality, taking its exponential and multiplying by $\left|\phi_{*}\left(t_{1}\right)\right|$ we come to (3.8). The question of an application of the equality (3.8) for establishing effective criteria of global solvability of eq (1.1) is an issue of separate study.

Using the lemma 2.4 in place of the lemma 2.3 by analogy it can be proved

Theorem 3.2. Let $\phi_{(0)} \neq 0, \frac{\phi_{(1)}}{\phi_{(0)}} \geq 0$, and let for some $\varepsilon>0$ the following inequalities hold:

$$
p_{0}(t ; w) \geq P(t), \quad q_{0}(t ; w) \geq Q(t) \geq 0, \quad r_{0}(t ; w) \leq 0, \quad\left|p_{0}(t ; w) \frac{r_{0}(t ; w)}{q_{0}(t ; w)}\right| \leq \widetilde{Q}(t)
$$

for $|w| \leq G_{M}\left(t_{0} ; t ; \phi_{(0)} ; p_{0}\left(t_{0} ; \phi_{(0)}\right) \frac{\phi_{(1)}}{\phi_{(0)}}\right)+\varepsilon, \quad t \geq t_{0}$. Then the solution $\phi_{0}(t)$ of eq. (1.1), satisfying the initial value conditions $\phi_{0}\left(t_{0}\right)=\phi_{(0)}, \phi^{\prime}\left(t_{0}\right)=\phi_{(1)}$, exists on the interval $\left[t_{0} ;+\infty\right)$. The function $\left|\phi_{0}(t)\right|$ is positive, nondecreasing and satisfies the inequality

$$
\left|\phi_{0}(t)\right| \leq G_{M}\left(t_{0} ; t ; \phi_{(0)} ; p_{0}\left(t_{0} ; \phi_{(0)}\right) \frac{\phi_{(1)}}{\phi_{(0)}}\right), \quad t \geq t_{0} .
$$

And if $\phi_{(1)} \neq 0$, then $\phi_{0}^{\prime}(t) \neq 0, \quad t \geq t_{0}$.

Theorem 3.3. Let $\phi_{1}(t)$ be a solution of eq. (2.8) on the interval $\left[t_{0} ;+\infty\right)$, and $\phi_{0}(t)$ be a solution of eq. (1.1) such, that

$\left.A_{1}\right) \phi_{1}\left(t_{0}\right) \geq \phi_{0}\left(t_{0}\right)>0, \phi_{1}^{\prime}\left(t_{0}\right)>\phi_{0}\left(t_{0}\right) \geq 0$ or

$$
\phi_{1}\left(t_{0}\right) \leq \phi_{0}\left(t_{0}\right)<0, \quad \phi_{1}^{\prime}\left(t_{0}\right)<\phi_{0}\left(t_{0}\right) \leq 0
$$

$\left.\mathrm{B}_{1}\right) p_{0}\left(t_{0} ; \phi_{0}\left(t_{0}\right)\right) \frac{\phi_{0}^{\prime}\left(t_{0}\right)}{\phi_{0}\left(t_{0}\right)}<p_{1}\left(t_{0} ; \phi_{1}\left(t_{0}\right)\right) \frac{\phi_{1}^{\prime}\left(t_{0}\right)}{\phi_{1}\left(t_{0}\right)}$.

Let in addition the following conditions hold:

$\left.\mathrm{C}_{1}\right) p_{0}(t ; w) \equiv p_{1}(t ; w)$ is a non increasing by $w$ on the interval $(-\infty ; 0]$ and non decreasing by $w$ on the interval $[0 ;+\infty)$ function;

$\left.\mathrm{D}_{1}\right) r_{1}\left(t ; w_{1}\right) \leq r_{0}(t ; w) \leq 0$ for $t \geq t_{0}, \quad|w| \leq\left|w_{1}\right|, \quad w, w_{1} \in(-\infty ;+\infty)$;

$\left.\mathrm{E}_{1}\right) \frac{q_{0}(t ; w)}{p_{0}(t ; w)} \leq \frac{q_{1}\left(t ; w_{1}\right)}{p_{1}\left(t ; w_{1}\right)}$ for $t \geq t_{0}, \quad|w| \leq\left|w_{1}\right|, \quad w, \quad w_{1} \in(-\infty ;+\infty)$.

Then $\phi_{0}(t)$ exists on the interval $\left[t_{0} ;+\infty\right)$, and the function $\left|\phi_{0}(t)\right|$ is positive and non decreasing.

Proof. Let $\left[t_{0} ; T\right)$ be the maximum interval of existence for $\phi_{0}(t)$. Let us show, that

$$
\phi_{0}(t) \neq 0, \quad t \in\left[t_{0} ; T\right) .
$$

Suppose, that this relation is false. Then it follows from $\left.A_{1}\right)$, that for some $T_{1} \in\left(t_{0} ; T\right)$

$$
\begin{gathered}
\phi_{0}(t) \neq 0, \quad t \in\left[t_{0} ; T_{1}\right) ; \\
\phi_{0}\left(T_{1}\right)=0 .
\end{gathered}
$$

It follows from $(3.10)$, that $y_{0}(t) \equiv p_{0}\left(t ; \phi_{0}(t)\right) \frac{\phi_{0}^{\prime}(t)}{\phi_{0}(t)}$ exists at least on the interval $\left[t_{0} ; T_{1}\right)$. It follows from $\left.\mathrm{A}_{1}\right)$, that $y_{0}(t) \geq 0, t \in\left[t_{0} ; T_{1}\right)$. By $(2.2)$ from here and $\left.\mathrm{A}_{1}\right)$ it follows: $\phi_{0}\left(T_{1}\right) \neq 0$, which contradicts (3.11). The obtained contradiction proves (3.9). It follows from (3.9), that $y_{0}(t)$ exists on the interval $\left[t_{0} ; T\right)$. Then since $y_{0}\left(t_{0}\right) \geq 0$, by virtue of $(2.5)$ it follows from $\left.\mathrm{D}_{1}\right)$, that

$$
y_{0}(t) \geq 0, \quad t \in\left[t_{0} ; T\right) .
$$


Since by condition of the theorem $\phi_{1}(t) \neq 0, t \geq t_{0}, y_{1}(t) \equiv p_{1}\left(t ; \phi_{1}(t)\right) \frac{\phi_{1}^{\prime}(t)}{\phi_{1}(t)}$ exists on the interval $\left[t_{0} ;+\infty\right)$. It follows from $\left.\mathrm{B}_{1}\right)$, that $y_{1}\left(t_{0}\right)>y_{0}\left(t_{0}\right)$. On the strength of lemma 2.5 it follows from here, $\left.\mathrm{A}_{1}\right)$ and $\left.\mathrm{C}_{1}\right)-\mathrm{E}_{1}$ ), that

$$
y_{0}(t)<y_{1}(t), \quad t \in\left[t_{0} ; T\right) .
$$

Let us show, that $T=+\infty$. Suppose $T<+\infty$. Then from $\left.B_{1}\right)$ and (3.13) it follows:

$$
\int_{t_{0}}^{T} \frac{y_{0}(\tau)}{p_{0}\left(\tau ; \phi_{0}(\tau)\right)} \mathrm{d} \tau \leq \int_{t_{0}}^{T} \frac{y_{1}(\tau)}{p_{0}(\tau ; 0)} \mathrm{d} \tau<+\infty .
$$

Using lemma 2.2 from here we conclude, that $\left[t_{0} ; T\right)$ is not maximum interval of existence for $y_{0}(t)$. But in the other hand since $\left[t_{0} ; T\right)$ is the maximum interval of existence for $\phi_{0}(t)$, then $\left[t_{0} ; T\right)$ is the maximum interval of existence for $y_{0}(t)$. We came to the contradiction. The obtained contradiction shows, that $T=+\infty$. Thus, $\phi_{0}(t)$ exists on the interval $\left[t_{0} ;+\infty\right)$. Due to $(2.2)$ it follows from $\left.A_{1}\right)$ and $(3.12)$, that the function $\left|\phi_{0}(t)\right|$ is positive and nondecreasing. The proof of the theorem is complete.

Definition 3.1. We call a solution $\phi_{0}(t)$ of eq. (1.1) singular oscillatory of second kind, if the existence domain of the $\phi_{0}(t)$ is a bounded set, and if $\phi_{0}(t)$ infinitely many times changes its sign.

Theorem 3.4. Let the following conditions hold:

$\left.\mathrm{A}_{2}\right) p_{0}(t ; w) \geq P(t), \quad \frac{q_{0}(t ; w)}{p_{0}(t ; w)} \geq Q(t), \quad t \geq t_{0}, \quad w \in(-\infty ;+\infty) ;$

$\left.\mathrm{B}_{2}\right) r_{0}(t ; w) \geq 0, \quad t \geq t_{0}, \quad w \in(-\infty ;+\infty)$,

Then for each $\phi_{(0)}$ and $\phi_{(1)}$ a non-extendable on the interval $\left[t_{0} ;+\infty\right)$ solution $\phi_{0}(t)$ of eq. (1.1), satisfying the initial value conditions $\phi_{0}\left(t_{0}\right)=\phi_{(0)}, \phi_{0}^{\prime}\left(t_{0}\right)=\phi_{(1)}$, is singular oscillatory of second kind.

Proof. Let $\left[t_{0} ; T\right) \quad(T<+\infty)$ be the maximum interval of existence for $\phi_{0}(t)$. Then, it is evident, that

$$
\sup _{\xi \in\left[T_{1} ; T\right)}\left|\phi_{0}(\xi)\right|>0, \quad T_{1} \in\left[t_{0} ; T\right) .
$$

(otherwise $\phi_{0}(t)$ will be extended by zero, i. e. $\phi_{0}(t) \equiv 0$ ) Let us show, that for each $T_{1} \in\left[t_{0} ; T\right)$ the function $\phi_{0}(t)$ has a zero on the interval $\left[T_{1} ; T\right)$. Suppose, that for some $T_{0} \in\left[t_{0} ; T\right)$ the function $\phi_{0}(t)$ has no zero on the interval $\left[T_{0} ; T\right)$. Let then $\phi_{0}(t)>0, t \in$

$\in\left[T_{0} ; T\right)$ (the proof in the case $\phi_{0}(t)<0, t \in\left[T_{0} ; T\right)$, by analogy). By (2.6) it follows from here, from $\mathrm{A}_{2}$ ) and $\mathrm{B}_{2}$ ), that

$$
\phi_{0}(t) \leq \frac{p_{0}\left(T_{0} ; \phi\left(T_{0}\right)\right)}{P(t)}\left|\phi^{\prime}\left(T_{0}\right)\right| \exp \left\{-\int_{T_{0}}^{t} Q(\tau) \mathrm{d} \tau\right\}, \quad t \in\left[T_{0} ; T\right) .
$$

Therefore, $\phi_{0}(t)$ is bounded. Then due to (2.6) and (2.7) there exists finite limits $\lim _{t \rightarrow T-0} \phi_{0}(t), \lim _{t \rightarrow T-0} \phi_{0}^{\prime}(t)$. It follows from here, that $\left[t_{0} ; T\right)$ is not maximum interval of existence for $\phi_{0}(t)$. The obtained contradiction shows, that for every $T_{1} \in\left[t_{0} ; T\right)$ the function $\phi_{0}(t)$ has a zero on the interval $\left[T_{1} ; T\right)$. On the basis of lemma 2.7 we conclude that from here, from $\left.\mathrm{B}_{2}\right)$ and $(3.14)$ it follows, that $\phi_{0}(t)$ is a singular oscillatory solution of second kind. The proof of the theorem is complete.

Definition 3.2. We call a solution of eq. (1.1) oscillatory, if it exists on the interval $\left[t_{0} ;+\infty\right)$ and in every neighborhood of $+\infty$ changes its sign.

Definition 3.3. We call a solution $\phi(t)$ of eq. (1.1) singular oscillatory of first kind, if it exists on the interval $\left[t_{0} ;+\infty\right)$, supp $\phi(t)$ is bounded and $\phi(t)$ infinitely many times changes its sign.

Let for every $\varepsilon>0$ the functions $p_{\varepsilon}(t), q_{\varepsilon}(t)$ and $r_{\varepsilon}(t)$ be real valued and continuous on the interval $\left[t_{0} ;+\infty\right)$, and let $p_{\varepsilon}(t)>0, t \geq t_{0}, \quad \varepsilon>0$. Consider the family of equations.

$$
\left(p_{\varepsilon}(t) \phi^{\prime}(t)\right)^{\prime}+q_{\varepsilon}(t) \phi^{\prime}(t)+r_{\varepsilon}(t) \phi(t)=0, \quad t \geq t_{0}, \quad \varepsilon>0 .
$$

Theorem 3.5. Let the following conditions hold:

$\left.\mathrm{A}_{3}\right) r(t ; w) \geq 0, \quad t \geq t_{0}, w \in(-\infty ;+\infty)$; 
$\left.\mathrm{B}_{3}\right)$ there exists $\varepsilon_{0}>0$ such, that for every $\varepsilon \in\left(0 ; \varepsilon_{0}\right]$

$$
p_{0}(t ; w) \leq p_{\varepsilon}(t), \frac{q_{0}(t ; w)}{p_{0}(t ; w)} \leq \frac{q_{\varepsilon}(t)}{p_{\varepsilon}(t)}, \quad r_{0}(t ; w) \geq r_{\varepsilon}(t), \text { for }|w| \geq \varepsilon, \quad t \geq t_{0},
$$

and eq. $\left(3.15_{\varepsilon}\right)$ is oscillatory;

$\mathrm{C}_{3}$ ) there exists $N>0$ such, that

$\left.\mathrm{C}_{3}^{1}\right) \quad p_{0}(t ; w) \leq P(t), \quad \frac{q_{0}(t ; w)}{p_{0}(t ; w)} \leq Q(t)$ for $|w| \leq N, \quad t \geq t_{0}$ and

$$
\int_{t_{0}}^{+\infty} \exp \left\{-\int_{t_{0}}^{\tau} Q(s) \mathrm{d} s\right\} \frac{\mathrm{d} \tau}{P(\tau)}=+\infty
$$

$\left.\mathrm{C}_{3}^{2}\right)$ for every $\varepsilon \geq N$ the following inequalities hold: $p_{0}(t ; w) \leq p_{\varepsilon}(t), \quad \frac{q_{0}(t ; w)}{p_{0}(t ; w)} \leq q_{\varepsilon}(t)$, $r_{0}(t ; w) \geq r_{\varepsilon}(t)$ for $N \leq|w| \leq \varepsilon$ and

$$
\int_{t_{0}}^{+\infty} \frac{\mathrm{d} \tau}{P(\tau)} \int_{t_{0}}^{\tau} \exp \left\{-\int_{s}^{\tau} q_{\varepsilon}(\xi) \mathrm{d} \xi\right\} r_{\varepsilon}(s) \mathrm{d} s=+\infty
$$

Then each existing on the interval $\left[t_{0} ;+\infty\right)$ nontrivial solution of eq. (1.1) either oscillatory, or singular oscillatory of first kind.

Proof.Let $\phi_{0}(t)$ be a solution of eq. (1.1) such, that supp $\phi_{0}(t)$ is unbounded on the interval $\left[t_{0} ;+\infty\right)$. Let us show, that $\phi_{0}(t)$ has arbitrarily large zeros. Suppose, that it is not so, i. e. there exists $t_{1} \geq t_{0}$ such, that $\phi_{0}(t) \neq 0, t \geq t_{1}$. Let then $\phi_{0}(t)>0, t \geq t_{1}$ (the proof in the case $\phi_{0}(t)<0, t \geq t_{1}$ by analogy). Due to (2.6) it follows from $\mathrm{A}_{3}$ ), that there can be one of the following three cases.

a) $\phi_{0}^{\prime}(t) \geq 0, \quad t \geq t_{1}$;

$\beta)$ there exists $t_{2} \geq t_{1}$ such, that $\phi_{0}(t) \leq N, t \geq t_{2}, \phi_{0}^{\prime}\left(t_{2}\right)<0$;

$\gamma)$ there exists $t_{2} \geq t_{1}$ such, that $\phi_{0}(t) \geq N, t \geq t_{2}, \quad \phi_{0}^{\prime}\left(t_{2}\right)<0$.

Let the case $\alpha$ ) holds, and let $0<\varepsilon<\min \left\{\phi_{0}\left(t_{1}\right) ; \varepsilon_{0}\right\}$. Then $y_{0}(t) \equiv p_{0}\left(t ; \phi_{0}(t)\right) \frac{\phi_{0}^{\prime}(t)}{\phi_{0}(t)}$ is a solution of eq. (2.1) on the interval $\left[t_{2} ;+\infty\right)$, and $y_{0}(t) \geq 0, t \geq t_{1}$. By virtue of lemma 2.6 it follows from here and from $\left.\mathrm{B}_{3}\right)$, that the Riccati's equation

$$
y^{\prime}(t)+\frac{y^{2}(t)}{p_{\varepsilon}(t)}+\frac{q_{\varepsilon}(t)}{p_{\varepsilon}(t)} y(t)+r_{\varepsilon}(t)=0, \quad t \geq t_{1},
$$

has a solution on the interval $\left[t_{1} ;+\infty\right)$. Consequently, corresponding equation $\left(3.15_{\varepsilon}\right)$ is not oscillatory, which contradicts $\left.\mathrm{C}_{3}\right)$. The obtained contradiction shows, that $\phi_{0}(t)$ has arbitrary large zeroes. Let the condition $\beta$ ) holds. Then it follows from $\mathrm{C}_{3}^{1}$ ), that

$$
\int_{t_{2}}^{t} \exp \left\{-\int_{t_{2}}^{\tau} \frac{q_{0}\left(s ; \phi_{0}(s)\right)}{p_{0}\left(s ; \phi_{0}(s)\right)}\right\} \frac{\mathrm{d} \tau}{p_{0}\left(\tau ; \phi_{0}(\tau)\right)} \geq \int_{t_{2}}^{t} \exp \left\{-\int_{t_{2}}^{\tau} Q(s) \mathrm{d} s\right\} \frac{\mathrm{d} \tau}{P(\tau)}, \quad t \geq t_{2}
$$

It is easy to show, that from (3.16) it follows equality $\int_{t_{2}}^{+\infty} \exp \left\{-\int_{t_{2}}^{\tau} Q(s) \mathrm{d} s\right\} \frac{\mathrm{d} \tau}{P(\tau)}=+\infty$. By (2.7) from here, from $\left.\mathrm{A}_{3}\right)$ and (3.18) it follows: $\lim _{t \rightarrow+\infty} \phi_{0}(t)=-\infty$, which contradicts the suggestion: $\phi_{0}(t)>0, t \geq t_{1}$. The obtained contradiction shows, that $\phi_{0}(t)$ has arbitrary large zeroes. Let the case $\gamma$ ) holds. Then from $\mathrm{C}_{3}^{2}$ ) it follows:

$$
\int_{t_{2}}^{t} \frac{\mathrm{d} \tau}{p_{0}\left(\tau ; \phi_{0}(\tau)\right)} \int_{t_{2}}^{\tau} \exp \left\{-\int_{s}^{\tau} \frac{q_{0}\left(\xi ; \phi_{0}(\xi)\right)}{p_{0}\left(\xi ; \phi_{0}(\xi)\right)} \mathrm{d} \xi\right\} r_{0}\left(s ; \phi_{0}(s)\right) \phi_{0}(s) \mathrm{d} s \geq
$$




$$
\geq \int_{t_{2}}^{t} \frac{\mathrm{d} \tau}{p_{\varepsilon}(\tau)} \int_{t_{2}}^{\tau} \exp \left\{-\int_{s}^{\tau} q_{\varepsilon}(\xi)\right\} r_{\varepsilon}(\tau) \mathrm{d} \tau
$$

By (2.7) from here and from(3.17) it follows: $\lim _{t \rightarrow+\infty} \phi_{0}(t)=-\infty$, which contradicts the suggestion: $\phi_{0}(t)>0, t \geq t_{1}$. The obtained contradiction shows, that $\phi_{0}(t)$ has arbitrary large zeroes. Thus, we showed, that a solution of eq. (1.1), existing on the interval $\left[t_{0} ;+\infty\right)$ and supp of which is an unbounded set, has arbitrary large zeroes. Due to lemma 2.7 it follows from here and from $\left.\mathrm{A}_{3}\right)$, that $\phi_{0}(t)$ is oscillatory.

Let $\phi_{0}(t)$ be a finite solution of eq. (1.1) on the interval $\left[t_{0} ;+\infty\right)$, and let $\phi_{0}(t)=$ $=0, \quad t \geq T$

$$
\max _{\xi \in\left[t_{1} ; T\right]}\left|\phi_{0}(\xi)\right|>0, \quad t_{1} \in\left[t_{0} ; T\right) .
$$

Let us show, that for each $T_{1} \in\left[t_{0} ; T\right)$ the function $\phi_{0}(t)$ has a zero on the interval $\left[T_{1} ; T\right)$. Suppose, that it is not so, i .e. there exists $T_{0} \in\left[t_{0} ; T\right)$ such, that $\phi_{0}(t)$ does not vanish on the interval $\left[T_{0} ; T\right)$. Since $\phi_{0}(T)=0$, on the strength of Lagrange's mean value theorem there exists $\xi \in\left[T_{0} ; T\right)$ such, that $\phi_{0}^{\prime}(\xi) \neq 0$, and sign $\phi_{0}\left(T_{0}\right)=-$ sign $\phi_{0}^{\prime}(\xi)$. By $(2.6)$ it follows from here and from $\left.\mathrm{A}_{3}\right)$, that $\phi_{0}^{\prime}(T) \neq 0$. But, in the other hand, since $\phi_{0}(t)=0$ for $t \geq T$, we have $\phi_{0}^{\prime}(T)=0$. We came to the contradiction. Consequently, for every $T_{1} \in\left[t_{0} ; T\right)$ the function $\phi_{0}(t)$ has a zero on the interval $\left[T_{1} ; T\right)$. Due to lemma 2.7 it follows from here, from $\left.A_{3}\right)$ and (3.19), that $\phi_{0}(t)$ is a singular oscillatory solution of first kind for eq. (1.1). The proof of the theorem is complete.

Remark 3.2. If the solution $\phi_{0}(t)$ of eq. (1.1), satisfying the initial value conditions $\phi_{0}\left(t_{0}\right)=\phi_{0}^{\prime}\left(t_{0}\right)=0$ is unique, $\left(\phi_{0}(t) \equiv 0\right)$, in particular, if $p_{0}(t ; w), q_{0}(t ; w)$ and $r_{0}(t ; w)$ satisfy the conditions of the remark 1.1, then eq. (1.1) has no singular oscillatory solutions of first kind.

Theorem 3.6. Let the conditions hold:

$\left.\mathrm{A}_{4}\right) r_{0}(t ; w) \geq 0$ for $t \geq t_{0}, w \in(-\infty ;+\infty)$

$\left.\mathrm{B}_{4}\right) p_{0}(t ; w), \frac{q_{0}(t ; w)}{p_{0}(t ; w)}, \quad-r_{0}(t ; w)$ are non increasing by $w$ on the interval $(-\infty ; 0]$ and nondecreasing by $w$ on the interval $[0 ;+\infty)$ functions.

Then for each $\phi_{(0)}$ and $\phi_{(1)}$ the solution $\phi_{0}(t)$ of eq. (1.1), satisfying the initial value conditions:

$$
\phi_{0}\left(t_{0}\right)=\phi_{(0)}, \quad \phi_{0}^{\prime}\left(t_{0}\right)=\phi_{(1)},
$$

exists on the interval $\left[t_{0} ;+\infty\right)$.

Proof. Let $\phi_{0}(t)$ be a solution of eq. (1.1), satisfying the initial value conditions $(3.19)$, and let $\left[t_{0} ; T\right)$ be the maximum interval of existence for $\phi_{0}(t)$. We should show, that $T=+\infty$. Suppose $T<+\infty$. Two cases are possible:

$\alpha)$ there exists $t_{1} \in\left[t_{0} ; T\right)$ such, that $\phi_{0}(t) \neq 0, \quad t \in\left[t_{1} ; T\right)$;

$\beta)$ there exists infinite sequence $t_{0}<t_{0}<t_{2}<\ldots<T$ such, that $\lim _{k \rightarrow+\infty} t_{k}=T, \phi_{0}\left(t_{k}\right)=0, k=1,2, \ldots$

Let the case $\alpha$ ) holds, and let $\phi_{0}(t)>0, t \in\left[t_{1} ; T\right.$ ) (the proof in the case $\phi_{0}(t)<0, t \in\left[t_{1} ; T\right.$ ), by analogy). If $\phi_{0}^{\prime}(t) \leq 0$, then by virtue of (2.7) it follows from $\left.\mathrm{A}_{4}\right)$, that $\phi_{0}(t)$ has finite (nonnegative) limit when $t \rightarrow T-0$. Then by virtue of (2.6) the function $\phi_{0}^{\prime}(t)$ also has finite limit when $t \rightarrow T-0$. Therefore, $\phi_{0}(t)$ continues to right at $T$, so $\left[t_{0} ; T\right)$ is not maximum interval of existence for $\phi_{0}(t)$. The obtained contradiction shows, that $T=+\infty$. Let $\phi_{0}^{\prime}\left(t_{1}\right)>0$. Two subcases are possible:

$\left.\alpha_{1}\right) \phi_{0}^{\prime}(t) \geq 0, \quad t \in\left[t_{1} ; T\right)$

$\left.\beta_{1}\right) \phi_{0}^{\prime}(t) \geq 0$, for $t \in\left[t_{1} ; T_{1}\right], \quad \phi_{0}^{\prime}(t)<0$, for $t \in\left(T_{1} ; T\right)$, for some $T_{1} \in\left(t_{1} ; T\right)$ (by virtue of $(2,2)$ and

$(2.5)$ it follows from $\left.\mathrm{A}_{4}\right)$, that if $\phi_{0}^{\prime}\left(\widetilde{t}_{1}\right)<0$ for some $\widetilde{t}_{1} \in\left(t_{1} ; T\right]$, then $\left.\phi_{0}^{\prime}(t)<0, t \in\left[\widetilde{t}_{1} ; T\right]\right)$.

In the case $\left.\alpha_{1}\right)$ the function $\phi_{0}(t)$ is nondecreasing on the interval $\left(t_{1} ; T\right)$. Then $(2.6)$ it follows from $\mathrm{A}_{4}$ ) and $\mathrm{B}_{4}$ ), that

$$
\phi_{0}^{\prime}(t) \leq \phi_{0}^{\prime}\left(t_{1}\right) \exp \left\{-\int_{t_{1}}^{t} \frac{q_{0}\left(\tau ; \phi_{0}\left(t_{1}\right)\right)}{p_{0}\left(\tau ; \phi_{0}\left(t_{1}\right)\right)} \mathrm{d} \tau\right\}, \quad t \in\left[t_{1} ; T\right) .
$$

Therefore, $\phi_{0}(t)$ has a finite limit when $t \rightarrow T-0$. It is evident, that the same we have in the case $\left.\beta_{1}\right)$. Then by (2.6) $\phi_{0}^{\prime}(t)$ has finite limit when $t \rightarrow T-0$. So, $\left[t_{0} ; T\right)$ is not maximum interval of existence for $\phi_{0}(t)$. The obtained contradiction shows, that $T=+\infty$. Let the case $\left.\beta\right)$ takes place. If $\phi_{0}(t)$ is bounded, 
then, it is evident, that the functions $\frac{1}{p_{0}\left(t, \phi_{0}(t)\right)}, \frac{q_{0}\left(t, \phi_{0}(t)\right)}{p_{0}\left(t, \phi_{0}(t)\right)}, r_{0}\left(t, \phi_{0}(t)\right)$ are bounded in the interval $\left[t_{0} ; T\right)$. By virtue of (2.7) it follows from here, that $\phi_{0}(t)$ has finite limit when $t \rightarrow T-0$. Then arguing similarly to the above (when we are dealing with the cases $\alpha_{1}$ ) and $\left.\beta_{1}\right)$ ) we conclude, that $T=+\infty$. Let $\phi_{0}(t)$ be not bounded. Two subcases are possible:

$\left.\alpha_{2}\right) \varlimsup_{t \rightarrow T-0} \phi_{0}(t)=+\infty$;

$\left.\beta_{2}\right) \underset{t \rightarrow T-0}{\stackrel{\lim }{\rightarrow T}} \phi_{0}(t)=-\infty$.

Let the case $\alpha_{2}$ ) holds (the proof in the case $\beta_{2}$ ) by analogy). Let $\phi_{1}(t)$ be the solution of eq. (1.1) with $\phi_{1}(T)=1, \quad \phi_{1}^{\prime}(T)=-1$. Then there exists $\zeta \in\left[t_{1} ; T\right)$ such, that $\phi_{1}(t)$ exists on the interval $[\zeta ; T]$, and

$$
\phi_{1}(t)>0, \quad \phi_{1}^{\prime}(t)<0, \quad t \in[\zeta ; T] .
$$

It follows from $\beta$ ) and $\left.\alpha_{2}\right)$, that there exists a point $\zeta_{1}(\in[\zeta ; T))$ of local maximum for $\phi_{0}(t)$ such, that $\phi_{0}\left(\zeta_{1}\right)>\max _{\xi \in[\zeta ; T]} \phi_{1}(\xi)$ and $\zeta<t_{m}<\zeta_{1}$ for some $m$. Since $t_{m}<\zeta_{1}<t_{n}$ for some $n>m$ and $\phi_{0}\left(t_{m}\right)=\phi_{0}\left(t_{n}\right)=0<\min \left\{\phi_{1}\left(t_{m}\right), \phi_{1}\left(t_{n}\right)\right\}$, but $\phi_{0}\left(\zeta_{1}\right)>\max \left\{\phi_{1}\left(t_{m}\right), \phi_{1}\left(t_{n}\right)\right\}$, there exist $\xi_{1}$ and $\xi_{2}$ such, that $\phi_{0}\left(\xi_{k}\right)=\phi_{1}\left(\xi_{k}\right), \quad k=1,2$ and

$$
\phi_{0}(t)>\phi_{1}(t), \quad t \in\left(\xi_{1} ; \xi_{2}\right) .
$$

(see pict. 1). Let $y_{j}(t) \equiv p_{0}\left(t ; \phi_{j}(t) \frac{\phi_{j}^{\prime}(t)}{\phi_{j}(t)}, j=0,1\right.$. Then by virtue of $\left(2.12_{1}\right)$ the following equality holds:

$$
\begin{aligned}
& y_{1}(t)-y_{0}(t)=\left[y_{1}\left(\zeta_{1}\right)-y_{0}\left(\zeta_{1}\right)\right] \exp \left\{-\int_{\zeta_{1}}^{t} \frac{y_{0}(\tau)+y_{1}(\tau)+q_{0}\left(\tau ; \phi_{0}(\tau)\right)}{p_{0}\left(\tau ; \phi_{0}(\tau)\right)} \mathrm{d} \tau\right\}- \\
& -\int_{\zeta_{1}}^{t} \exp \left\{-\int_{\tau}^{t} \frac{y_{0}(s)+y_{1}(s)+q_{0}\left(s ; \phi_{0}(s)\right)}{p_{0}\left(s ; \phi_{0}(s)\right)} \mathrm{d} s\right\}\left[\left(\frac{1}{p_{1}\left(\tau ; \phi_{1}(\tau)\right)}-\frac{1}{p_{0}\left(\tau ; \phi_{0}(\tau)\right)}\right) y_{1}^{2}(\tau)+\right. \\
& \left.\quad+\left(\frac{q_{1}\left(\tau ; \phi_{1}(\tau)\right)}{p_{1}\left(\tau ; \phi_{1}(\tau)\right)}-\frac{q_{0}\left(\tau ; \phi_{0}(\tau)\right)}{p_{0}\left(\tau ; \phi_{0}(\tau)\right)}\right) y_{1}(\tau)+r_{1}\left(\tau ; \phi_{1}(\tau)\right)-r_{0}\left(\tau ; \phi_{0}(\tau)\right)\right] \mathrm{d} \tau=0 .
\end{aligned}
$$

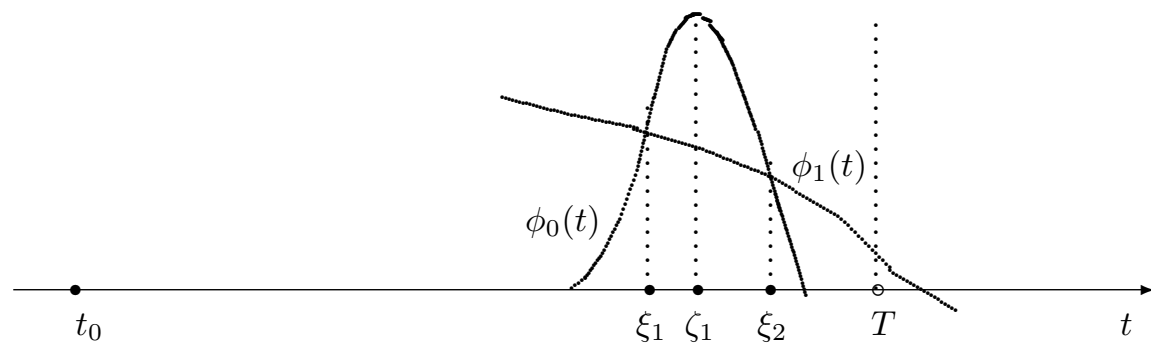

pict. 1

An illustration to the part of proof of Theorem 3.6, related to the case $\left.\alpha_{2}\right)$. Here $\left[t_{0} ; T\right)$ is the maximum existence interval for $\phi_{0}(t)$. The "cap" in this picture is a part of the graph of $\phi_{0}(t)$.The decreasing curve is a part of the graph of $\phi_{1}(t) ; \xi_{1}$ and $\xi_{2}$ are points of intersection of graph of $\phi_{0}(t)$ and $\phi_{1} ; \zeta_{1}$ is a local maximum point of $\phi_{0}(t)$.

It is evident, that

$$
y_{1}\left(\zeta_{1}\right)<y_{0}\left(\zeta_{1}\right)(=0)
$$

Since $\phi_{0}(t) \geq \phi_{1}(t)>0$ on the interval $\left[\zeta_{1} ; \xi_{2}\right]$, then it follows from the conditions of the theorem, that

$$
p_{0}\left(t ; \phi_{0}(t)\right) \geq p_{0}\left(t ; \phi_{1}(t)\right), \quad r_{0}\left(t ; \phi_{0}(t)\right) \leq r_{0}\left(t ; \phi_{1}(t)\right), \frac{q_{0}\left(t ; \phi_{0}(t)\right)}{p_{0}\left(t ; \phi_{0}(t)\right)} \geq \frac{q_{0}\left(t ; \phi_{1}(t)\right)}{p_{0}\left(t ; \phi_{1}(t)\right)}, \quad t \in\left[\zeta_{1} ; \xi_{2}\right] .
$$


Then since $y_{1}(t)<0$ on the interval $\left[\zeta_{1} ; \xi_{2}\right]$, it follows from $(3.23)$ and $(3.24)$, that $y_{1}\left(\xi_{2}\right)<y_{0}\left(\xi_{2}\right)$. Then $\phi_{1}^{\prime}\left(\xi_{2}\right)<\phi_{0}^{\prime}\left(\xi_{2}\right)$. It follows from here, that $\phi_{1}^{\prime}(t)<\phi_{0}^{\prime}(t), t \in\left[\xi_{3} ; \xi_{2}\right]$, for some $\xi_{3} \in\left(\zeta_{1} ; \xi_{2}\right)$. Therefore, $\int_{\xi_{3}}^{\xi_{2}}\left(\phi_{1}(\tau)-\phi_{0}(\tau)\right)^{\prime} \mathrm{d} \tau<0$, or, which is the same, $\phi_{1}\left(\xi_{3}\right)>\phi_{0}\left(\xi_{3}\right)$ (since $\phi_{1}\left(\xi_{2}\right)=\phi_{0}\left(\xi_{2}\right)$, see pict.1), which contradicts (3.22). The obtained contradiction shows, that $T=+\infty$. The proof of the theorem is complete.

\section{Some Applications}

Consider the Emden - Fowler equation (see [2], p. 171):

$$
\left(t^{\rho} \phi^{\prime}(t)\right)^{\prime}-t^{\sigma} \phi^{n}(t)=0, \quad t \geq t_{0}>0, \quad n>1, \quad \rho, \quad \sigma \in(-\infty ;+\infty) .
$$

Along with this equation, consider the equation

$$
\left(t^{\rho} \phi^{\prime}(t)\right)^{\prime}-t^{\sigma}|\phi(t)|^{n-1} \phi(t)=0, \quad t \geq t_{0} .
$$

Here $p_{0}(t ; w) \equiv t^{\rho}, q_{0}(t ; w) \equiv 0, \quad r_{0}(t ; w) \equiv-t^{\sigma}|w|^{n-1}$. We put: $P(t) \equiv t^{\rho}, Q(t) \equiv$ $\equiv 0, R(t) \equiv-t^{\sigma}$. Let $\rho>1$. Then

$$
F\left(t_{0} ; t ; c_{1} ; c_{2}\right) \leq\left|c_{1}\right| \exp \left\{\frac{c_{2}}{\rho-1}\left(t_{0}^{1-\rho}-t^{1-\rho}\right)+\frac{t^{\sigma+2-\rho}-t_{0}^{\sigma+2-\rho}}{(\sigma+1)(\sigma+2-\rho)}-\frac{t_{0}^{\sigma+2-\rho}}{(\sigma+1)(\rho-1)}\right\} .
$$

It follows from this, that if $-1<\sigma<\rho-1$, then

$$
F\left(t_{0} ; t ; c_{1} ; c_{2}\right) \leq\left|c_{1}\right| \exp \left\{\frac{c_{2}}{\rho-1} t_{0}^{1-\rho}-\frac{t_{0}^{\sigma+2-\rho}}{(\sigma+1)(\sigma+2-\rho)}\right\} \stackrel{\text { def }}{=} A\left(t_{0} ; c_{1} ; c_{2}\right),
$$

and if $\sigma<-1$, then

$$
F\left(t_{0} ; t ; c_{1} ; c_{2}\right) \leq\left|c_{1}\right| \exp \left\{\frac{c_{2}}{\rho-1} t_{0}^{1-\rho}-\frac{t_{0}^{\sigma+2-\rho}}{(\sigma+1)(\rho-1)}\right\} \stackrel{\text { def }}{=} B\left(t_{0} ; c_{1} ; c_{2}\right) .
$$

It is not difficult to see, that if

$$
-1<\sigma<\rho-2, \quad A\left(t_{0} ; \phi_{(0)} ; t_{0}^{\rho} \frac{\phi_{(1)}}{\phi_{(0)}}\right)<1,
$$

or

$$
\sigma<-1, \quad B\left(t_{0} ; \phi_{(0)} ; t_{0}^{\rho} \frac{\phi_{(1)}}{\phi_{(0)}}\right)<1,
$$

then for eq. (4.2) the conditions of the theorem 3.1 hold. Therefore, for every $\phi_{(0)}, \phi_{(1)}$, satisfying the conditions $\phi_{(0)} \neq 0, \frac{\phi_{(1)}}{\phi_{(0)}} \geq 0$ and one of the conditions (4.3) or (4.4), the solution $\phi_{0}(t)$ of eq. (4.2) with $\phi_{0}\left(t_{0}\right)=\phi_{(0)}, \phi_{0}^{\prime}\left(t_{0}\right)=\phi_{(1)}$, exists on the interval $\left[t_{0} ;+\infty\right)$, and if takes place $(4.3)$, then

$$
\left|\phi_{0}(t)\right| \leq A\left(t_{0} ; \phi_{(0)} ; t_{0}^{\rho} \frac{\phi_{(1)}}{\phi_{(0)}}\right), \quad t \geq t_{0},
$$

and if takes place (4.4), then

$$
\left|\phi_{0}(t)\right| \leq B\left(t_{0} ; \phi_{(0)} ; t_{0}^{\rho} \frac{\phi_{(1)}}{\phi_{(0)}}\right), \quad t \geq t_{0} ;
$$

the function $\left|\phi_{0}(t)\right|$ is positive and nondecreasing. It is evident, that if $\phi_{(0)}>0$, then $\phi_{0}(t)$ is a solution of eq. (4.1) too. 
Remark 4.1. If $\phi(t)$ is a positive (negative) solution of eq. (4.2) (and $n=\frac{n_{1}}{n_{2}}$, where $n_{1}$ and $n_{2}$ are odd), then $\phi(t)$ is a solution of eq. (4.1) too. Note that equations (4.1) and (4.2) are not equivalent for all $n$, e. g. the equation $\phi^{\prime \prime}(t)-\frac{1}{t} \phi^{2}(t)=0$ is not equivalent to equation $\phi^{\prime \prime}(t)-\frac{1}{t}|\phi(t)| \phi(t)=0$, since the function $\phi_{0}(t) \equiv-\frac{2}{t}$ is a solution of the last equation on the interval $[1 ;+\infty)$, but not of the first one.

Let $\rho=0, \quad \sigma+n+1<0$. Then (see [2], p. 173) the function $\phi_{B}(t) \equiv\left[\frac{(\sigma+2)(\sigma+n+1)}{(n-1)^{2}}\right]^{\frac{1}{n-1}} t^{-\frac{\sigma+2}{n-1}}$ is a solution of eq. (4.2). It is not difficult to see, that for $p(t ; w)=p_{1}(t ; w) \equiv 1, q(t ; w)=q_{1}(t ; w) \equiv$ $0, \quad r(t ; w)=r_{1}(t ; w)=-t^{\sigma}|w|^{n-1}, \quad t \geq t_{0}, w \in(-\infty ;+\infty), \quad \rho=0, \quad \sigma+n+1<0$ for equations (2.8)and (4.2) the conditions $\left.\left.\mathrm{C}_{1}\right)-\mathrm{E}_{1}\right)$ of the theorem 3.3 hold. Therefore, the solution $\phi_{0}(t)$ of eq. (4.2) with $\phi_{0}\left(t_{0}\right) \neq 0, \frac{\phi_{0}^{\prime}\left(t_{0}\right)}{\phi_{0}\left(t_{0}\right)} \leq \frac{\phi_{B}^{\prime}\left(t_{0}\right)}{\phi_{B}\left(t_{0}\right)}$, exists on the interval $\left[t_{0} ;+\infty\right)$, and $\left|\phi_{0}(t)\right|$ is positive and nondecreasing. It is evident that if $\phi_{0}\left(t_{0}\right)>0$, then $\phi_{0}(t)$ is a solution of eq. (4.1). For $\phi_{0}\left(t_{0}\right)<0$ the function $\phi_{0}(t)$ will be a solution of eq. (4.1) if $n=\frac{n_{1}}{n_{2}}$, where $n_{1}$ and $n_{2}$ are odd. If $\rho \neq 1$, then by invertible transformation

$$
\left\{\begin{array}{l}
s=(\rho-1)^{-1} t^{\rho-1}, \quad \phi=(\rho-1)^{(\rho-\sigma-2) /[(\rho-1)(n-1)]} \frac{\psi}{s} \quad \text { for } \rho>1 \\
s=(1-\rho)^{-1} t^{1-\rho}, \quad \phi=(1-\rho)^{-(\sigma+\rho) /[(n-1)(1-\rho)]} \psi, \quad \text { for } \rho<1 .
\end{array}\right.
$$

eq. (4.2) converts in (see [2], pp. 171, 172)

$$
\psi^{\prime \prime}(s)-s^{\sigma_{1}}|\psi(s)|^{n-1} \psi(s)=0, \quad s \geq s_{0}>0,
$$

where

$$
\sigma_{1}=\left\{\begin{array}{l}
\frac{\sigma+\rho}{\rho-1}-(n+3), \quad \rho>1 \\
\frac{\sigma+\rho}{1-\rho}, \quad \rho<1
\end{array}\right.
$$

This transformation establishes a one-to-one correspondence between solutions of equations (4.2) and (4.7), and existing on the interval $\left[t_{0} ;+\infty\right)$ solutions of eq. (4.2) transform in solutions of eq. (4.7), existing on the interval $\left[s_{0} ;+\infty\right)$. By already proved above for $\sigma_{1}+n+1<0$ eq. (4.7) has two - parameter family of solutions on the interval $\left[s_{0} ;+\infty\right)$. Therefore from (4.8) it follows, that in the cases $\rho>\max \{1, \sigma+2\}$ and $\frac{\sigma-1}{n}+1<\rho<1$ eq. (4.2) has two - parameter family of solutions on the interval $\left[t_{0} ;+\infty\right)$.

Definition 4.1. We say, that a solution $\left(\phi_{0}(t), \psi_{0}(t)\right)$ of eq. (1.2) is conditionally stable for $t \rightarrow+\infty$, if there exists one dimensional manifold $S \ni\left(\phi_{0}\left(t_{0}\right), \psi_{0}\left(t_{0}\right)\right)$ such, that for every $\varepsilon>0$ and for every solution $(\phi(t), \psi(t))$ of the system (1.2) there exists $\delta>0$ such, that $\left|\phi(t)-\phi_{0}(t)\right|+\left|\psi(t)-\psi_{0}(t)\right|<\varepsilon$ for $t \geq t_{0}$, as soon as $\left(\phi\left(t_{0}\right), \psi\left(t_{0}\right)\right) \in S$ and $\left|\phi\left(t_{0}\right)-\phi_{0}\left(t_{0}\right)\right|+\left|\psi\left(t_{0}\right)-\psi_{0}\left(t_{0}\right)\right|<\delta$ (see [10], p. 314).

Definition 4.2. We say, that a solution $\phi_{0}(t)$ of eq. (1.1) is conditionally stable for $t \rightarrow+\infty$, if the corresponding solution $\left(\phi_{0}(t), p_{0}\left(t ; \phi_{0}(t)\right) \phi_{0}^{\prime}(t)\right)$ of the system (1.2) is conditionally stable for $t \rightarrow+\infty$.

Let us show, that if $\rho>1, \sigma<-1$, then the solution $\phi_{0}(t) \equiv 0$ of eq. (4.1) is conditionally stable for $t \rightarrow+\infty$. Let $S=\left\{\left(\phi_{(0)}, \phi_{(1)}\right): 0 \leq \phi_{(0)} \leq \exp \left\{\frac{t_{0}^{\sigma+2-\rho}}{(\sigma+1)(\rho-1)}\right\}, \phi_{(1)}=0\right\}$, and let $\phi(t)$ be a solution of eq. (4.1) with $\left(\phi\left(t_{0}\right), t_{0}^{\rho} \phi_{0}^{\prime}\left(t_{0}\right)\right) \in S, \phi\left(t_{0}\right) \neq 0$. Then $B\left(t_{0} ; \phi\left(t_{0}\right) ; 0\right)<1$. By virtue of theorem 3.1 it follows from here and from (4.4), that $\phi(t)$ exists on the interval $\left[t_{0} ;+\infty\right)$ and satisfies the inequality

$$
|\phi(t)| \leq \phi\left(t_{0}\right) \exp \left\{-\frac{t_{0}^{\sigma+2-\rho}}{(\sigma+1)(\rho-1)}\right\}, \quad t \geq t_{0}>0 .
$$

By virtue of (4.1) it follows from her, that

$$
\left|t^{\rho} \phi^{\prime}(t)\right| \leq \phi\left(t_{0}\right) \exp \left\{-\frac{t_{0}^{\sigma+2-\rho}}{(\sigma+1)(\rho-1)}\right\}\left(\frac{-t_{0}^{\sigma+1}}{\sigma+1}\right), \quad t \geq t_{0}>0 .
$$

Let $\varepsilon>0$ be fixed. We put:

$$
\delta=\delta(\varepsilon) \equiv \frac{\varepsilon}{2}\left(1-\frac{t_{0}^{\sigma+1}}{\sigma+1}\right)^{-1} \exp \left\{\frac{t_{0}^{\sigma+2-\rho}}{(\sigma+1)(\rho-1)}\right\}
$$


Let $\left|\phi\left(t_{0}\right)\right|+\left|t_{0}^{\rho} \phi^{\prime}\left(t_{0}\right)\right|<\delta$. Then it follows from (4.9) and (4.10), that

$$
|\phi(t)|+\left|t_{0}^{\rho} \phi^{\prime}(t)\right|<\varepsilon \text { for } t \geq t_{0}
$$

If $\phi\left(t_{0}\right)=0$, then by virtue of remark $1.1 \phi(t) \equiv 0$, and, therefore, in this case the relation (4.11) also takes place. Consequently, the solution $\left(\phi_{0}(t), \psi_{0}(t)\right) \equiv(0,0)$ of the system

$$
\left\{\begin{array}{l}
\phi^{\prime}(t)=\frac{\psi(t)}{t^{\rho}} ; \\
\psi^{\prime}(t)=t^{\sigma} \phi^{n}(t)
\end{array}\right.
$$

is conditionally stable for $t \rightarrow \infty$. Then the solution $\phi_{0}(t) \equiv 0$ of eq. (4.1) is conditionally stable for $t \rightarrow \infty$. Taking into account remark 4.1 we summarize the obtained result in the following form.

Theorem 4.1. The following assertions hold.

I). Let $\rho>1$, and let $\phi_{(0)}$ and $\phi_{(1)}$ satisfy the conditions: $\phi_{(0)} \neq 0, \frac{\phi_{(1)}}{\phi_{(0)}} \geq 0$ and one of the conditions (4.3), (4.4). Then the solution $\phi_{0}(t)$ of eq. (4.2), satisfying the initial value conditions: $\phi_{0}\left(t_{0}\right)=\phi_{(0)}, \phi_{0}^{\prime}\left(t_{0}\right)=$ $\phi_{(1)}$, exists on the interval $\left[t_{0} ;+\infty\right)$. The function $\left|\phi_{0}(t)\right|$ is positive and nondecreasing, and if $(4.3)$ holds, then the estimate (4.5) is valid, and if (4.4) holds, then the estimate (4.6) is valid. If $\phi_{(0)}>0$ or if $\phi_{(0)}<0, \quad n=\frac{n_{1}}{n_{2}}$, where $n_{1}$ and $n_{2}$ are odd, then $\phi_{0}(t)$ is a solution of (4.1).

II). Let $\rho=0, \sigma+n+1<0$. Then the solution $\phi_{0}(t)$ of eq. (4.2) with $\phi_{0}\left(t_{0}\right) \neq 0,0 \leq \frac{\phi_{0}^{\prime}\left(t_{0}\right)}{\phi_{0}\left(t_{0}\right)}<\frac{\phi_{B}^{\prime}\left(t_{0}\right)}{\phi_{B}\left(t_{0}\right)}$, exists on the interval $\left[t_{0} ;+\infty\right)$, and $\left|\phi_{0}(t)\right|$ is positive and nondecreasing. If $\phi_{0}\left(t_{0}\right)>0$ or if $\phi_{0}\left(t_{0}\right)<$ $0, n=\frac{n_{1}}{n_{2}}$, where $n_{1}$ and $n_{2}$ are odd, then $\phi_{0}(t)$ is a solution of eq. (4.1).

III). In the cases $\rho>\max \{1, \sigma+2\}$ and $\frac{\sigma-1}{n}+1<\rho<1$ eq. (4.1) has two - parameter family of solutions on the interval $\left[t_{0} ;+\infty\right)$.

IV). If $\rho>1$ and $\sigma<-1$, then $\phi_{0}(t) \equiv 0$ of eq. (4.1) is conditionally stable for $t \rightarrow+\infty$.

Remark 4.2. In the case $\rho=0$ the existence of global solutions of eq. (4.1), which are different by their properties from described in assertion II of theorem 4.1 (the Kneser's solutions) follows from theorem 16.1 of book [1] (see [1], p. 371).

Let us compare theorem 4.1 with the following result (see [11], p. 8).

Theorem*. The following assertions hold:

i). There exists $\varepsilon>0$ such, that every solution of eq. (4.7) with Cauchy initial conditions $|\psi(s)| \leq$ $\varepsilon, \mid \psi^{\prime}\left(s \mid \leq \varepsilon\right.$ exists on the interval $\left[s_{0} ;+\infty\right)$ if and only if $\sigma_{1}<-n-1$.

ii). If $\sigma_{1} \geq-n-1$, then every solution $\psi(s)$ of eq. (4.7), satisfying $\psi(\tau) \psi^{\prime}(\tau)>0$ at some $\tau \geq s_{0}$ is non continuable on the interval $\left[s_{0} ;+\infty\right)$.

In the assertions $I)$ and $I I)$ of the theorem 4.1 the region of the initial values $\phi\left(t_{0}\right), \phi^{\prime}\left(t_{0}\right)\left(\psi\left(s_{0}\right), \psi^{\prime}\left(s_{0}\right)\right)$ for which the solution $\phi(t)(\psi(s))$ of eq. (4.2) (of eq. (4.7)) exists on the interval $\left[t_{0} ;+\infty\right)\left(\left[s_{0} ;+\infty\right)\right)$ is describes by well - defined relationships, whereas from the assertion i) of the theorem* we can not see exactly for which initial conditions $\psi\left(s_{0}\right), \psi^{\prime}\left(s_{0}\right)$ (except the trivial case $\left.\psi\left(s_{0}\right)=\psi^{\prime}\left(s_{0}\right)=0\right)$ the solution $\psi(s)$ of eq. (4.7) exists on the interval $\left[s_{0} ;+\infty\right)$. From the assertion ii) of the theorem* it follows, that in the assertion II) of the theorem 4.1 the condition $\sigma+n+1<0$ can not be replaced by weaker condition $\sigma+n+1 \leq 0$. In this sense theorem 3.3 (which implies II)) is sharp. From the assertion ii) of theorem* and from (4.8) it follows, that in the assertion III) of theorem 4.1 the condition $\rho>\max \{1, \sigma+2\}$ $\left(\frac{\sigma-1}{n}+1<1\right)$ can not be replaced by weaker condition $\rho \geq \max \{1, \sigma+2\}\left(\frac{\sigma-1}{n}+1 \leq 1\right)$. In this sense theorem 3.1 (which is used in proof of III)) is sharp.

Let $\lambda(t), \mu(t)$ and $\nu(t)$ be continuous functions on the interval $\left[t_{0} ;+\infty\right)$ and let $\lambda(t)>0, \mu(t) \geq$ $0, \nu(t) \geq 0, \quad t \geq t_{0}$. Consider the following Van der Pol's type equation (see [12]).

$$
\left(\lambda(t) \phi^{\prime}(t)\right)^{\prime}+\mu(t)\left(\phi^{2}(t)-1\right) \phi^{\prime}(t)+\nu(t) \phi(t)=0, \quad t \geq t_{0} .
$$

Here $p_{0}(t ; w) \equiv \lambda(t), q_{0}(t ; w) \equiv \mu(t)\left(w^{2}-1\right), \quad r_{0}(t ; w) \equiv \nu(t)$ satisfy all of the conditions of theorem 3.6. Therefore, for each $\phi_{(0)}$ and $\phi_{(1)}$ the solution $\phi_{0}(t)$ of eq. (4.12), satisfying the initial value conditions: $\phi_{0}\left(t_{0}\right)=\phi_{(0)}, \quad \phi_{0}^{\prime}\left(t_{0}\right)=\phi_{(1)}$, exists on the interval $\left[t_{0} ;+\infty\right)$. We put:

$$
P(t)=p_{\varepsilon}(t) \equiv \lambda(t), \quad Q(t) \equiv 0, \quad q_{\varepsilon}(t)=\mu(t)\left(\varepsilon^{2}-1\right), r_{\varepsilon}(t)=\nu(t),
$$


$t \geq t_{0}, \varepsilon>0, N=1$. It is not difficult to check, that if the following conditions hold:

$\left.\mathrm{a}^{\circ}\right) . \int_{t_{0}}^{+\infty} \frac{\mathrm{d} \tau}{\lambda(\tau)}=\int_{t_{0}}^{+\infty} \frac{\mathrm{d} \tau}{\lambda(\tau)} \int_{t_{0}}^{\tau} \exp \left\{-\int_{s}^{\tau} \frac{\mu(\xi)\left(\varepsilon^{2}-1\right)}{\lambda(\xi)}\right\} \nu(s) \mathrm{d} s=+\infty$ when $\varepsilon \geq N$;

$\left.\mathrm{b}^{\circ}\right)$. for $0<\varepsilon \leq \varepsilon_{0}$ the equations

$$
\left(\lambda(t) \phi^{\prime}(t)\right)^{\prime}+\mu(t)\left(\varepsilon^{2}-1\right) \phi^{\prime}(t)+\nu(t) \phi(t)=0, \quad t \geq t_{0}
$$

are oscillatory, then for eq. (4.12) with (4.13) the conditions $\mathrm{A}_{3}$ ) - $\mathrm{B}_{3}$ ) of theorem 3.5 hold. Then due to theorem 3.5 the solution $\phi_{0}(t)$ either is oscillatory or is singular oscillatory of first kind. Since it is evident that the functions $p_{0}(t ; w) \equiv \lambda(t), \quad q_{0}(t ; w) \equiv \mu(t)\left(w^{2}-1\right), \quad r_{0}(t ; w) \equiv \nu(t)$ satisfy the conditions of the remark 1.1, the solution $\phi_{0}(t)$, satisfying the initial value conditions $\phi_{0}\left(t_{0}\right)=\phi_{(0)}, \phi_{0}^{\prime}\left(t_{0}\right)=\phi_{(1)}$, is exactly one, and consequently, due to remark 3.2 cannot be singular oscillatory of first kind. The obtained result we summarize in the following form.

Theorem 4.2. Let $\lambda(t)>0, \mu(t) \geq 0, \nu(t) \geq 0$ for $t \geq t_{0}$. Then for each $\phi_{(0)}$ and $\phi_{(1)}$ the solution $\phi_{0}(t)$ of eq. (4.12), satisfying the initial value conditions:

$\phi_{0}\left(t_{0}\right)=\phi_{(0)}, \phi_{0}^{\prime}\left(t_{0}\right)=\phi_{(1)}$, exists on the interval $\left[t_{0} ;+\infty\right)$. Moreover if in addition the conditions $\left.\mathrm{a}^{\circ}\right)$ and $\mathrm{b}^{\circ}$ ) hold, then $\phi_{0}(t)$ is oscillatory.

\section{Conclusion}

We have used the Riccati equation method to investigate some classes of second order nonlinear ordinary differential equations. This method have made possible us to establish four new global existence criteria for the mentioned classes of equations. We have proved two new oscillatory criteria for them as well. These criteria were used to the Emden - Fowler equation, having applications in the astrophysics, and to the Wan der Pole type equation, which is applicable for studying the dynamics of dusty grain charge in dusty plasmas.

\section{References}

1. Kiguradze I. T., Chanturia T. A. The asymptotic behavior of solutions of nonlinear ordinary differential equations, Moscow, "Nauka", 1990.

2. Bellman R. Stability theory of differential equations, Moscow, Izdatelstvo inostrannoj literatury, 1954 (New York, Toronto, London, McGRAW-HILL BOOK COMPANY, INC, 1053).

3. Hartman Ph. Ordinary differential equations. Moscow, "Mir", 1970 (JOHN WILLEY and SONS, New York - London - Sydney 1064).

4. Reising R., Sansone G. Conty R. Qualitative theory of nonlinear differential equations, Moscow, "Nauka", 1974 (EDIZIONI CREMONESE ROMA 1065).

5. Jordan D. W., Smith P. Nonlinear differential equations. Oxford University press, 2007.

6. Cheng Y., Peng M., Zhang W. On the asymptotic behavior of the solutions of a class of second order nonlinear differential equations. Journal of Computational and Applied Mathematics 98 (1998) pp. 63 79 .

7. Motohiko K., Kusano T. On a class of second order quasilinear ordinary differential equations. Hiroshima Math. J., v. 25, 1995, pp. 321 - 355.

8. Kusano T., Akio O. Existence and asymptotic behavior of positive solutions of second order quasilinear differential equations. Funkcialax Ekvacioj, v. 37, 1994, pp. 345 - 361.

9. Grigorian G. A. On two comparison tests for second-order linear ordinary differential equations (Russian) Differ. Uravn. 47 (2011), no. 9, 1225 - 1240; translation in Differ. Equ. 47 (2011), no. 91237 - 1252, 34 C10. 10. Demidovich B. P. Lectures on the mathematical stability theory, Moscow, "Nauka", 1967.

11. Pekarkova E. Asymptotic Properties of Second Order Differential Equations With p - Laplacian (Dissertation). Brno, Masarik University, Mathematics and Statistics, 2009.

12. Momeni M., Kurakis I., Mosley - Fard M., Shukla P. K. A Van der Pol - Mathieu equation for the dynamics of dust grain charge in dusty plasmas. J. Phys, A: Math.Theor., v. 40, 2007, F473 - F481. 